\title{
Implications of the Sharpe Ratio as a Performance Measure in Multi-Period Settings
}

\author{
Jakša Cvitanić, Ali Lazrak and Tan Wang ,
}

May 15, 2007

\begin{abstract}
We study effects of using Sharpe ratio as a performance measure for compensating money managers in a dynamic and frictionless market setting. First, we demonstrate that with such a performance measure, the manager's focus on the short horizon performance is detrimental to the investor's long horizon performance. Numerical experiments illustrate that when returns are iid, the performance loss is significant, even when the investor's investment horizon is not much longer than the manager's. When expected returns are mean reverting, the performance loss is exacerbated. Second, we show that Sharpe ratio maximization strategies tend to increase (decrease) the risk in the later part of the optimization period after a bad (good) performance in the earlier part of the optimization period. As illustrated by a simulation exercise, this prediction is in agreement with empirical observations, and it presents a rational expectations alternative to the prevalent tournament theory explanation.

Keywords: Sharpe Ratio, Performance Measures, Money Managers.
\end{abstract}

* Jakša Cvitanić (corresponding author) is with the California Institute of Technology, Division of The Humanities and Social Sciences, M/C 228-77 Caltech 1200 E. California Blvd. Pasadena, CA 91125. E-mail: cvitanic@hss.caltech.edu; Ali Lazrak is with the Sauder School of Business at the University of British Columbia, Vancouver, BC, V6T 1Z2, E-mail: lazrak@sauder.ubc.ca. Tan Wang is with the Sauder School of Business at the University of British Columbia and CCFR, Vancouver, BC, V6T 1Z2, E-mail: tan.wang@sauder.ubc.ca. We would like to express our gratitude to Murray Carlson, Murray Frank, Emmanuel Gobet, Monique Jeanblanc, Alan Kraus as well as participants at the UBC finance seminar, the Asset Pricing and Microstructure conference IFM/HEC Montreal (2003), Bachelier World Congress Chicago (2004), NFA (2005), The workshop adavnces in portfolio decision making Notre Dame (2007) and The bank of Canada-Rotman workshop on advances in portfolio management Toronto (2007) for helpful discussions and comments. We are grateful to the editor and the anonymous referees for many suggestions. J. Cvitanić is grateful to the National Science Foundation (Grant NSF-DMS 04-03575) for support. Wang and Lazrak are grateful to the SSRHC and Lazrak is grateful to the NSERC. 


\section{Introduction}

Institutional portfolio management has significantly increased over the past four decades ${ }^{1}$. The risk-adjusted performance measurement is an important factor for fund investors when selecting fund managers, and for deciding on the ways for compensating them. Although introduced almost four decades ago, the Sharpe ratio (Sharpe $(1966,1994)$ and Modigliani and Modigliani (1997)), is still one of the most commonly used measures of the performance of money managers.

The aim of this paper is to report and quantify some implications of the dynamic investment strategy of a fund manager whose compensation is based on the Sharpe ratio performance measure (for instance in form of a quarterly bonus). More specifically, we compute and analyze, in a couple of different models, the portfolio strategy with the maximal Sharpe ratio of a manager who trades dynamically in a frictionless market.

Our first implication is that the Sharpe ratio creates a tension between the short term performance and the long term performance. This is what we call the horizon problem. To see this, assume that there is an investor who has the maximization of the Sharpe ratio as his objective for a given horizon. Assume that this investor invests with a mutual fund manager who also maximizes the Sharpe ratio, but who has a shorter horizon. The horizon problem simply points out that the manager's and the investor's interests are then not aligned. For example, a strategy that maximizes the one-year Sharpe ratio for ten years, will be different (and inferior for the overall horizon) to the strategy which maximizes the ten-year Sharpe ratio. In order to assess the practical importance of the horizon problem, we investigate quantitatively how much damage the interests of the long-term investor suffer in such a situation. We study this in two different settings. One is where the returns are iid. We show that in this setting, the difference between the optimal long-term Sharpe ratio and the suboptimal long-term Sharpe ratio that results from maximizing short-term Sharpe ratios, can be as large as $6 \%$ for a one-year investment horizon and over $40 \%$ for a 5 -year horizon. In the second setting, the returns follow a mean-reverting process. In this setting, there is predictability in expected returns and long-term risk is smaller (Barberis (2000), Campbell and Viciera (1999)). Thus, the longer investment horizon should allow the investor to take advantage of the predictability and the smaller risk. Indeed, we show that the difference in Sharpe ratio is even larger. For the parameters used in this paper, the difference in Sharpe

\footnotetext{
${ }^{1}$ According to the 2002 Mutual Fund Fact Book published by the Investment Company Institute, the total mutual fund industry net assets in US have grown from 17 billion dollars in 1960 to 6.97 trillion dollars in 2001, an over 400-fold increase. In the same period, the number of funds has increased from 361 to 8,307 .
} 
ratios for a two-year investment horizon are more than two times larger than in the iid environment. $^{2}$

Our second implication concerns the time series behavior of the Sharpe ratio maximizing investment strategy. We show that for a fixed horizon, say a year, the risk of the portfolio in the second half of the year is negatively related to the performance of the portfolio in the first half of the year. More precisely, after periods of high returns, the fund manager has the incentive to lower the risk, while after periods of low returns, the manager has the incentive to increase the risk. This prediction is very general: it does not require any specific assumptions on the returns dynamics, other than a complete market. It is also consistent with the empirical findings of Brown et al (1996) who show that the funds that have a poor half-year performance tend to increase the risk of their positions in the second half of the year ${ }^{3}$. They rationalize this finding with the so called "tournament view" of fund management. In this view, the fund managers compete with each other for the limited investment pool of the fund investors. The empirical literature documents that the fund investors are likely to invest in a fund which realizes a good performance at the end of the year ${ }^{4}$. Now, since the managers' compensation is typically proportional to the value of the assets under management, the managers have an incentive to improve their return towards the end of the year, often by taking unusually high risks. These incentives are even stronger when the mid-year performance has been bad (relative to the targeted expected returns). Our framework provides an alternative explanation for the empirical finding of Brown et al (1996): it is consistent with a standard, rational expectations model of portfolio choice where the managers maximize the Sharpe ratio, and it does not require the "tournament" explanation. To make this point concrete, we perform a numerical exercise where we assume that the manager maximizes the one year Sharpe ratio, and simulate 3000 paths of returns from the iid returns model. As in Brown et al (1996), we estimate the ratio of the risk exposure in the second period of the year and the risk exposure in the first period of the year (denoted $\left.\sigma_{2} / \sigma_{1}\right)$ and construct a $2 \times 2$ frequency table which presents the level of risk ratio $\left(\sigma_{2} / \sigma_{1}\right)$

\footnotetext{
${ }^{2}$ In the real world, it is of course difficult to identify clearly the investment horizon of the fund investors. However, it is reasonable to argue that more established funds have a longer investment horizon than younger funds. The result of our paper may be tested empirically along that dimension.

${ }^{3}$ There are still some mixed views on the issue: Busse (2001), argues that the result of Brown et al (1996) may be the result of a bias in their volatility estimates. Qiu (2003), using a different data set, found that the result of Brown et al does not hold for that data set.

${ }^{4}$ See, among others, Ippolito (1992), Berkowitz and Kotowitz (1993, 2000), Goetzmann and Peles (1997), Chevalier and Ellison (1997), Sirri and Tufano (1998), Koski and Pontiff (1999), Huang, Wei and Yan (2004). See also Berk and Green (2004) for a rational model of the relationship between the investment flows and the performance.
} 
relative to the interim mid-year performance. The direction of our results is systematically consistent with the Brown et al (1996) findings and the magnitude is comparable.

A substantial literature in financial economics addresses the performance measurement issue in general, and the Sharpe ratio in particular ${ }^{5}$. According to the mean-variance theory developed by Markowitz (1952) and the Capital Asset Pricing model developed by Sharpe (1964) and Lintner (1965), the portfolios with the highest Sharpe ratio are mean-variance efficient. While Sharpe ratio derives its appeal from its simplicity, its theoretical foundation relies on several restrictive assumptions. In particular, the efficiency property of Sharpe ratio requires either the assumption that investors have a mean-variance utility (Leland (1999), Bernardo and Ledoit (2000)), or an assumption on the distributional properties of the returns (for example, normal distribution). More recently, Sharpe ratio has also been studied under conditions of non-symmetrical distributions (Leland (1999)) and in the presence of derivative securities (Spurgin (2001) and Goetzmann, Ingersoll, Spiegel, and Welch (2002)). This recent literature shows that when out-of-the-money options can be written, a manager can enhance the Sharpe ratio of his portfolio by altering the tail of its return distribution. This is possible because Sharpe ratio depends only on the first two moments of the distribution of the portfolio return. By sacrificing the higher moments of the distribution, one can enhance the mean-variance tradeoff and hence obtain higher Sharpe ratios. This "manipulation" possibility presents a drawback of using the Sharpe ratio. Our work extends this literature in two directions. First, our framework emphasizes another drawback of the Sharpe ratio performance measure, namely the tension between the long term performance and the short term performance. Given the high pressure that mutual fund managers experience relative to the short term performance, the horizon problem is directly relevant for the mutual funds investors. Second, the form of the Sharpe ratio maximizing strategies that we compute, gives insight in how the managers would reallocate their portfolio if they wanted to manipulate the Sharpe ratio. For instance, our results suggest that in order to duplicate the short outof-the-money option positions reported in Goetzmann, Ingersoll, Spiegel, and Welch (2002), one has to use a dynamic investment strategy which induces a higher risk exposure after bad performance.

Let us mention here that we do not model in this paper "agency" (e.g. moral hazard) issues of delegated portfolio management, other than those arising from the difference in the time horizons of the investors and the managers. And even for this issue, we do not study how to reduce the inefficiencies arising from the horizon problem, we just compute their size in different models. There is a growing literature that examines explicitly the

\footnotetext{
${ }^{5}$ See Grinblatt and Titman (1995) for a review.
} 
agency issues in portfolio management, and its potential asset pricing implications. That literature typically searches for optimal contracts, or compares different types of contracts, but there are no horizon or other timing issues. Some more recent examples include OuYang (2003a,b), Cadenillas, Cvitanić and Zapatero (2004), and Cuoco and Kaniel (2003). As the objective of our paper is the study of the dynamic implications of the Sharpe ratio performance measure, we focus our attention on that, and abstract from other agency issues.

In terms of the mathematical methodology for the mean-variance problems in multiperiod settings, we do not offer new insights, as the theory has been thoroughly developed in recent years (most notably, using the theory of Backward Stochastic Differential Equations). This literature includes among others, the following papers: Korn and Trautmann (1995), Bajeux, Portait (1998), Li and Ng (2000), Zhou and Li (2000), Lim and Zhou (2002), Lim (2003), Bielecki, Pliska, Jin and Zhou (2005). A textbook presentation for the case of a single risky asset with constant parameters is available in Cvitanić and Zapatero (2004). As it is done in part of this literature, we assume a complete market, which enables us to use the so-called martingale approach, which is conceptually quite simple. In particular, the approach allows us to provide semi-explicit computations for the optimal strategy in the general complete market model (Proposition 3), and new, explicit computations in the special case of the model with mean reversion expected returns (Proposition 6).

The rest of the paper is organized as follows. Section 2 uses a simple binomial setting to illustrate the main economic implications of our study. Section 3 describes a continuoustime investment environment and the definition of Sharpe ratio and then provides the general solution to the Sharpe ratio maximization problem for different horizons. Section 4 studies quantitatively the difference in Sharpe ratios maximized by long-run and short-run money managers in an economy with a constant investment opportunity set. Section 5 studies the same issue when the stock price follows a mean-reverting process. Section 6 analyzes the time series properties of the Sharpe ratio maximizing strategies and performs a simulation exercise. Finally, Section 7 concludes. Technical details are collected in the appendix.

\section{A Simple Binomial Example}

In this section, we describe in a simple four-period binomial model the basic intuition behind our study. The mathematical form of the optimal portfolio could be obtained directly from a general discrete multi-period framework analyzed in $\mathrm{Li}$ and $\mathrm{Ng}$ (2000), but we choose to present this very simple example to point out the main economic insights. 
We will consider each period to be a quarter, and assume that the performance of a portfolio is reported every six months. In this model, the long-term investor desires the trading strategy that maximizes the Sharpe ratio of his portfolio for a one-year horizon, while the short-term manager chooses the portfolio that maximizes the six-month Sharpe ratio and renews the same procedure at the end of the second quarter.

Assume that each quarter the stock either increases by $u=10 \%$ or falls by $d=10 \%$ with probabilities $60 \%$ and $40 \%$, respectively. Let $z(t)$ denote the corresponding random return for quarter $t(t=1, . ., 4)$. Assume, for simplicity, that the risk free rate is $0 \%$.

Consider first the long-term investor with an initial wealth $X(0)$ and the investment horizon of four quarters. Let $\pi(t)$ be the dollar amount he has invested in the stock at time $t$. The wealth at time 1 is then

$$
X(1)=\pi(0) z(1)+(X(0)-\pi(0))=\pi(0)(z(1)-1)+X(0)
$$

and by recursion, the terminal wealth $X(4)$ associated with the strategy $\pi$ is given by

$$
X(4)=X(0)+\sum_{t=1}^{4}(z(t)-1) \pi(t-1)
$$

The long-term investor's objective is to choose a strategy that maximizes the Sharpe ratio of his portfolio over the four-quarter period:

Problem 1: Choose a portfolio strategy $\pi(t)$ to minimize $\operatorname{Var}(X(4))$ under the constraint $E(X(4)) \geq X(0)(1+b)^{4}$ where $b$ is an arbitrary, but positive level of expected return, which we fix at $2 \%$.

The problem is straightforward to solve. The optimal portfolio strategy is described by the left tree in Figure 1.

It gives the optimal portfolio weights, that is, the wealth proportion invested in the stock. The weights depend on the past returns. More specifically, after a good realized return, the weight decreases, while after a bad return, the weight increases. This dependence will be crucial for the understanding of the empirical implications of the Sharpe ratio maximizing strategy ${ }^{6}$.

The problem of the short-term manager is

Problem 2: At $t=0$, choose the investment strategy $(\pi(0), \pi(1))$ that minimizes $\operatorname{Var}(X(2))$ under the constraint $E[X(2)] \geq X(0)(1+b)^{2}$. Then, at $t=2$, choose the investment strategy

\footnotetext{
${ }^{6}$ Technically speaking, these are Markovian strategies, whose value can be determined from the current wealth value, but the latter indirectly depends on the past performance.
} 

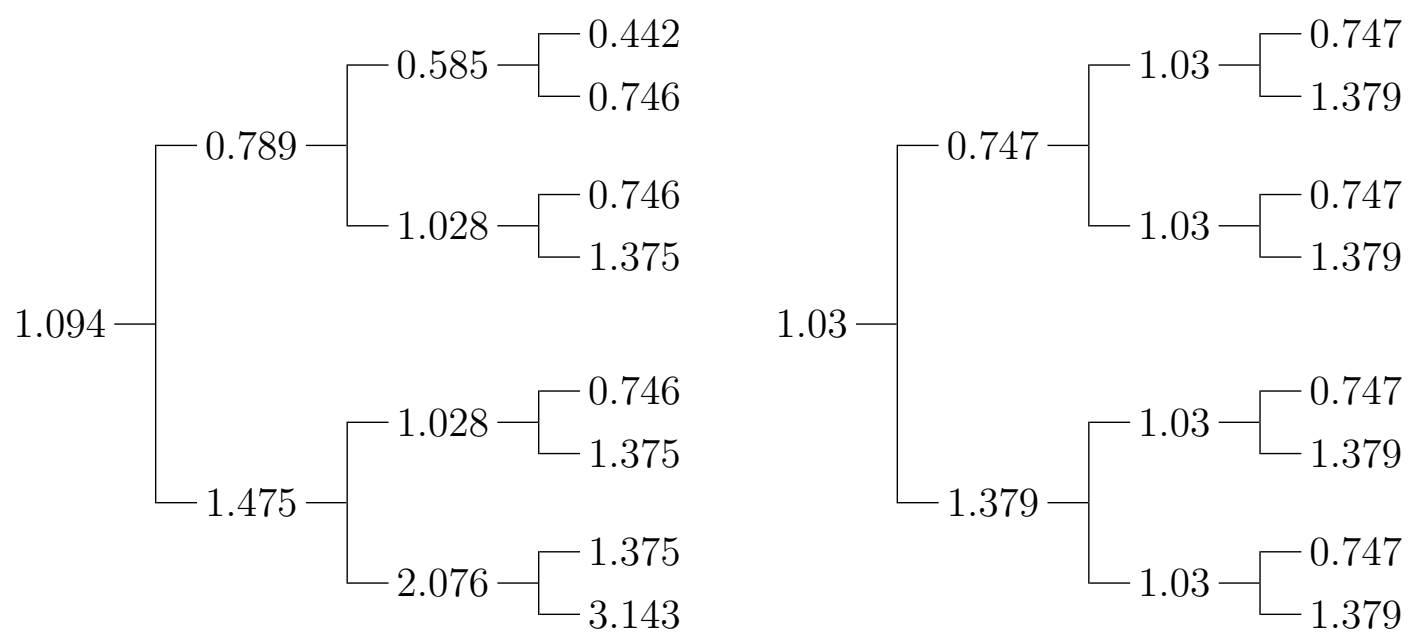

Figure 1: The Optimal Trading Strategy for the Long-term and the Short-term horizon The trees give the optimal portfolio weights in the stock, the left tree for the long horizon, and the right tree for the short horizon.

$(\pi(2), \pi(3))$ that minimizes $\operatorname{Var}_{2}(X(4))$ under the constraint $E_{2}[X(4)] \geq X(2)(1+b)^{2}$, where the variance $V \mathrm{rr}_{2}$ and the expectation $E_{2}$ are conditional on the information at time $t=2$.

Solving Problem 2 yields the optimal weights shown in the right tree in Figure 1. Like the optimal long-term strategy, the optimal short-term strategy is negatively correlated with the past returns. However, at the performance assessment date $t=2$, this effect is stopped, and then it restarts again at time $t=3$, because the manager restarts his optimization problem.

We make two observations based on this example. First, the solution to Problem 1 yields the optimal Sharpe ratio of 0.4212, while the solution to Problem 2 yields the optimal Sharpe ratio of 0.4027. The difference in the Sharpe ratios illustrates how the incentives of the longterm investor and of the short-term manager are not perfectly aligned, as mentioned in the introduction. Second, the optimal trading strategies of both problems are dependent on how good the past performance has been.

In order to illustrate the (straightforward) intuition for these effects, in Figure 2 we show the expected returns over the remaining periods implied by the optimal trading strategy. If the return history has been good (resp. bad) the remaining expected return is low (resp. high). Since the long-term strategy targets a $2 \%$ annual expected return over four quarters, a good return in the first quarter means that a lower expected return will be targeted in the coming quarters. This implies using a strategy with a lower risk in the coming quarters, 
which, in turn, brings down the overall risk of the portfolio. Conversely, after a bad return in the first quarter, the a higher expected return will be targeted for the following periods, which necessarily raises the risk of the portfolio. This adjustment of the strategy according to the past returns represents the dependence on the past performance mentioned above.

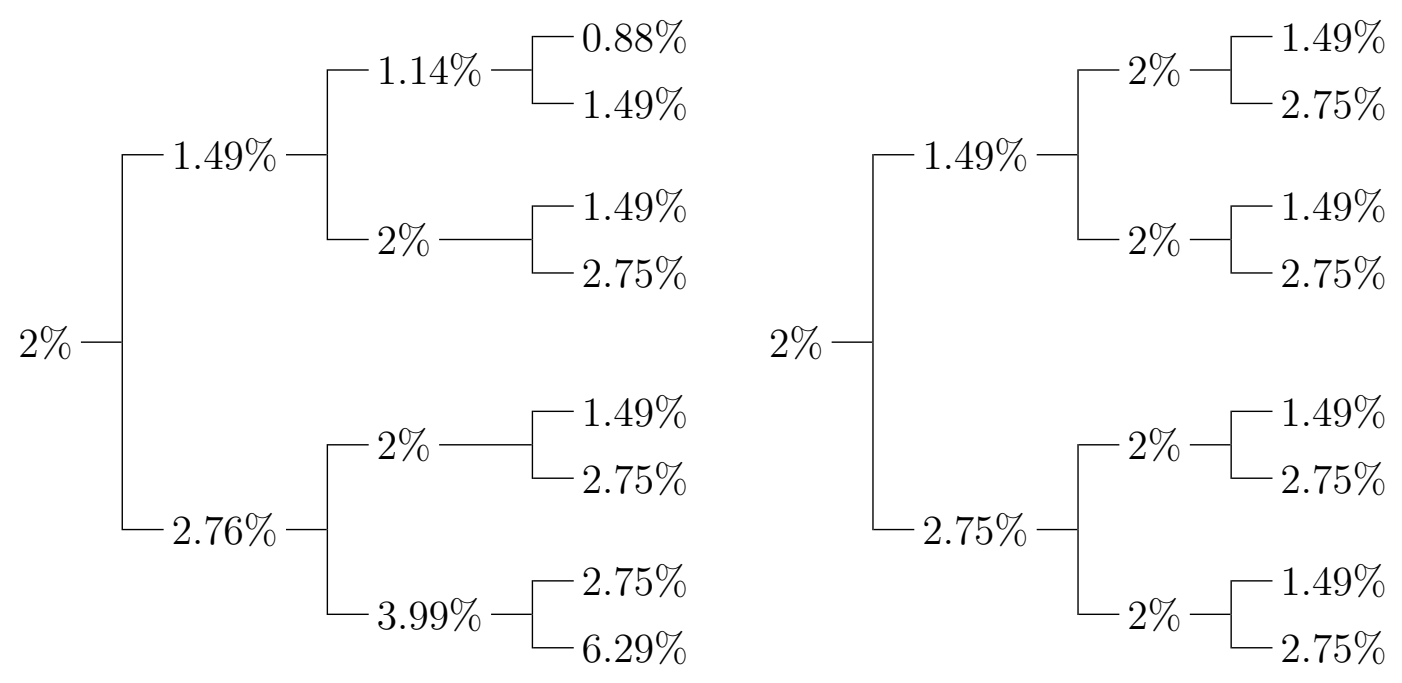

Figure 2: Remaining Expected Returns Implied by the Optimal Trading Strategies

The strategy which maximizes the Sharpe ratio for a given level of expected return should be contrasted with the strategy that maximizes a CRRA utility. In iid settings, which include the binomial model of this section, an agent with a CRRA utility would optimally set the portfolio weights to be constant, hence, in particular, horizon-invariant (see for example Merton (1971)). However, when the agent's objective function is quadratic,

$$
E\left[X_{\mathcal{T}}\right]-\frac{\gamma}{2} \operatorname{Var}\left(X_{\mathcal{T}}\right)
$$

corresponding to the problem of maximizing Sharpe ratio, this is no longer true, as we saw in the above example, and as we will see in the more elaborate models below. Such an agent follows a trading strategy that is indirectly influenced by the past performance and it depends also on the horizon, even though the investment opportunities are iid.

In the following sections, we will extend the analysis also to non-iid settings, with changing investment opportunities leading to more interesting stock holding dynamics. We will adopt a continuous-time framework, which will give us analytical tractability and allow us to obtain explicit solutions which are easily amenable to numerical calculations. 


\section{Sharpe Ratio in a Continuous Time Setting}

We begin by providing an expression for Sharpe ratio in a continuous time setting. Apart from completeness of the market, we impose as few restrictions as possible on the financial markets. The assumption of the market completeness is mainly for analytical convenience, as it allows us to derive closed-form solutions. These results can also be derived from the general theory developed in the papers mentioned at the end of the introduction.

The underlying uncertainty is modeled by the complete probability space $(\Omega, \mathcal{F}, P)$ endowed with a filtration $\mathcal{F}=\left\{\mathcal{F}_{t} ; \mathcal{T} \geq t \geq 0\right\}$ representing the information available to the investor up to time $\mathcal{T}$. This filtration is the usual $P$ - augmentation of the filtration generated by the $J$-dimensional Brownian motion $W(t)=\left(W_{1}(t), \ldots, W_{J}(t)\right)^{\top}$.

There are $J$ risky assets (stocks) indexed by $i \in\{1,2, \cdots, J\}$ and a riskless asset in the economy. The prices of these risky assets evolve according to the equations

$$
d S_{i}(t)=S_{i}(t)\left[\mu_{i}(t) d t+\sum_{j=1}^{J} \sigma_{i j}(t) d W_{j}(t)\right], \quad S_{i}(0)>0, i=1, \ldots, J,
$$

where the $J$-vector process $\mu(t)=\left(\mu_{1}(t), \cdots, \mu_{J}(t)\right)^{\top}$ represents the mean returns and the $J \times J$-matrix process $\sigma(t)=\left\{\sigma_{i j}(t)\right\}_{1 \leq i, j \leq J}$ represents co-variances of the returns. The riskless asset provides a constant interest rate $r$. We assume that $\sigma(t)$ is invertible almost surely. Thus the market is complete. The processes $\mu(t), \sigma(t)$, and $\sigma^{-1}(t)$ are all assumed to be progressively measurable with respect to $\mathcal{F}$ and bounded uniformly.

Let

$$
\beta(t) \equiv \exp \{-r t\}, \quad 0 \leq t
$$

and

$$
\theta(t) \equiv \sigma^{-1}(t)[\mu(t)-r \cdot \mathbf{1}]
$$

where $\mathbf{1}=(1,1, \ldots, 1)^{\top}$ is an $J$-vector of ones. Under the assumption made above, the process $Z(t)$ defined by

$$
Z(t)=\exp \left(-\int_{0}^{t} \theta^{\top}(s) d W(s)-\frac{1}{2} \int_{0}^{t} \theta^{\top}(s) \theta(s) d s\right),
$$

is a martingale and thus we may define the risk neutral probability measure by

$$
d \tilde{P}=Z(\mathcal{T}) d P
$$

By Girsanov Theorem, the $J$-dimensional process $\left(\tilde{W}_{1}(t), \cdots, \tilde{W}_{J}(t)\right)^{\top}$ defined by

$$
\tilde{W}(t) \equiv W(t)+\int_{0}^{t} \theta(s) d s, \quad t \leq \mathcal{T}
$$


is a Brownian motion under $\tilde{P}$. Under the risk neutral probability measure, the discounted stock prices $\beta(t) S(t)$ are martingales. Finally, we define

$$
\xi(t)=\beta(t) Z(t)
$$

which is the state-price density process.

We will assume that the objective of the manager is to maximize the Sharpe ratio of the portfolio under his management over $\mathcal{T}$ years. This of course corresponds to the standard Markowitz problem for the investment horizon $\mathcal{T}$. Let $x$ be the initial wealth under management. For simplicity, we assume that there is no additional influx or deduction of funds from the managed portfolio.

Denote by $X(t)$ the portfolio wealth value at time $t$. In other words, $X(t)$ is the value of a dollar invested at time zero. Denote by $\pi(t)$ the vector of amounts the manager invests in the risky assets. Then the value of the wealth evolves according to

$$
X(t)=x+\int_{0}^{t}\left(\sum_{i=1}^{J} \pi_{i}(s)\left(\mu_{i}(t)-r\right)+r X(s)\right) d s+\int_{0}^{t}\left(\sum_{i, j=1}^{J} \pi_{i}(s) \sigma_{i j}(s) d W_{j}(s)\right) .
$$

Note that the discounted wealth process satisfies

$$
\beta(t) X(t)=x+\int_{0}^{t}\left(\sum_{i, j=1}^{J} \beta(s) \pi_{i}(s) \sigma_{i j}(s) d \tilde{W}_{j}(s)\right),
$$

under the risk neutral measure. Thus, $\beta X$ is a $\tilde{P}$-martingale under mild regularity conditions. By definition, the set $\mathcal{A}(x)$ of admissible strategies consists of those portfolio processes $\pi$ for which $\beta X$ is a $\tilde{P}$ - martingale satisfying (5).

More formally, the objective of the manager is to minimize

$$
\operatorname{Var}(X(\mathcal{T}))
$$

subject to

$$
E(X(\mathcal{T}))=x e^{b \mathcal{T}}
$$

and to equation (4), where $b>r$ is an arbitrary, but fixed annualized expected return and $X(\mathcal{T})$ is the portfolio value at time $\mathcal{T}{ }^{7}$

\footnotetext{
${ }^{7}$ As mentioned in Introduction, the minimization problem (6) is the multi-period mean-variance problem studied in a number of papers in the mathematical finance literature (Korn and Trautmann (1995), Bajeux, Portait (1998), Li and Ng (2000), Zhou and Li (2000), Lim and Zhou (2002), Lim (2003), Bielecki, Pliska, Jin and Zhou (2005) among others). These papers develop a comprehensive theory for the problem and some of them provide closed form expressions for the optimal trading strategy in particular contexts. While the focus of this paper is different, we do add a contribution to this literature by solving for the optimal trading strategy when returns are mean reverting.
} 
In the real world, different funds may have different investment horizons, which may also differ from the horizon of the performance measurement period. For example, a typical performance measurement period is one year, while a more established fund may care more about the long run average performance and pay less attention to the year by year performance. ${ }^{8}$ We consider two types of funds, a short-run fund and a long-run fund. Let $\mathcal{T}$ denote the horizon in years of the long-run fund and $X_{L}(\mathcal{T})$ its wealth at time $\mathcal{T}$. We will assume that the investment horizon of the short-run fund coincides with that of the performance measurement period, $\tau$. This enables us to interpret the short-run fund as the fund which is completely aligned with the interests of the manager who cares only about the next performance measurement.

Let $N=\mathcal{T} / \tau$, assumed to be an integer, for simplicity. At the beginning of every quarter $T_{n}$, the short-run manager's investment objective is

$$
\inf _{\pi \in \mathcal{A}\left(X_{S}\left(T_{n}\right)\right)} \operatorname{Var}_{T_{n}}\left(X_{S}\left(T_{n+1}\right)\right) \equiv E\left[\left(X_{S}\left(T_{n+1}\right)-E\left[X_{S}\left(T_{n+1}\right) \mid \mathcal{F}_{T_{n}}\right]\right)^{2} \mid \mathcal{F}_{T_{n}}\right]
$$

subject to

$$
E\left[X_{S}\left(T_{n+1}\right) \mid \mathcal{F}_{T_{n}}\right]=X_{S}\left(T_{n}\right) e^{b \tau}
$$

where $X_{S}\left(T_{n}\right)$ is the portfolio wealth at time $T_{n}$ and $\mathcal{A}\left(X_{S}\left(T_{n}\right)\right)$ is the set of feasible investment strategies for the time period $\left(T_{n}, T_{n+1}\right)$.

Note that while the objectives of both the short-run and long-run managers are the maximization of Sharpe ratio, the investment horizons differ, one being $\mathcal{T}$ years and the other being $\tau$. The following proposition provides the general expression of the maximal Sharpe ratio for an investment horizon $T$, and versions of this result are available in the literature mentioned at the end of Introduction and in Footnote 7.

Proposition 1 Let $x$ be the initial value of the fund. Denote

$$
\Lambda(t, s)=E\left[(Z(s) / Z(t))^{2} \mid \mathcal{F}_{t}\right]=\frac{E\left[Z^{2}(s) \mid \mathcal{F}_{t}\right]}{Z^{2}(t)}
$$

Then the maximum value of Sharpe ratio

$$
\mathcal{S}_{T}=\frac{x\left(e^{b T}-e^{r T}\right)}{\sqrt{\operatorname{Var}(X(T))}}
$$

\footnotetext{
${ }^{8}$ As indicated in Introduction, and illustrated by results in this section and later sections, the best expected year-by-year performance may not give the best expected performance over a longer horizon, in terms of Sharpe ratio.
} 
for investment horizon $T$ is given by ${ }^{9}$

$$
\mathcal{S}_{T}=\sqrt{\Lambda(0, T)-1}
$$

In particular, this maximum Sharpe ratio is independent of $b$.

Denote by

$$
\mathcal{S}_{L}=\frac{x\left(e^{b \mathcal{T}}-e^{r \mathcal{T}}\right)}{\sqrt{\operatorname{Var}\left(X_{L}(\mathcal{T})\right)}} \equiv \sqrt{\Lambda(0, \mathcal{T})-1}
$$

the maximum long-run Sharpe ratio. The short-run manager maximizes the $\tau$-year Sharpe ratio. Thus if $X_{S}\left(T_{n}\right)$ is the value of the fund after $n$ periods at the beginning of the $n \tau$ year, then the value of the fund after another period will be $X_{S}\left(T_{n+1}\right)$ where $X_{S}\left(T_{n+1}\right)$ is the result of the optimization problem $(8)$. Let $X_{S}(\mathcal{T})$ be the value of the fund at time $\mathcal{T}=T_{N}$ as the result of period by period Sharpe ratio maximization by the short-run fund manager. In other words, $X_{S}(\mathcal{T})$ is the wealth of an investor's who invest $x$ at time zero, has the long horizon $\mathcal{T}$, but whose portfolio is managed by the short-term manager. As we will verify in the proof of Corollary 2, the short-term manager achieves the return $b$ on the long horizon, that is, $E\left(X_{S}(\mathcal{T})\right)=x e^{b \mathcal{T}}$, so that the Sharpe ratio of the short term manager on the long horizon is given by

$$
\mathcal{S}_{S} \equiv \frac{x\left(e^{b \mathcal{T}}-e^{r \mathcal{T})}\right.}{\sqrt{\operatorname{Var}\left(X_{S}(\mathcal{T})\right)}}
$$

Since $\mathcal{S}_{L}$ maximizes Sharpe ratio on the long horizon, it is to be expected that $\mathcal{S}_{L} \geq \mathcal{S}_{S}$. In fact, since $\mathcal{S}_{L}$ is independent of $b$, we have $\mathcal{S}_{L} \geq \mathcal{S}_{S}$, no matter what target $b$ the short-run manager has. Thus, we have the following result:

Corollary 2 Assume that the short-run manager sets an arbitrary, but fixed expected target return $b>r$ for all periods. If $N>1$, then the risk of the portfolio managed by the short-run manager is higher than that of the long-run manager, no matter what the value of $b>r$ is. That is, $\operatorname{Var}\left(X_{S}(\mathcal{T})\right) \geq \operatorname{Var}\left(X_{L}(\mathcal{T})\right)$, and, consequently, $\mathcal{S}_{L} \geq \mathcal{S}_{S}$, for all $b>r$.

Thus, the optimal Sharpe ratio that can be achieved on a long horizon $\mathcal{T}$ is always at least as high than that of the Sharpe ratio achieved by a short-run manager, no matter what the value of the targeted return is (actually, as we illustrate below, it is usually strictly higher). A prediction of this corollary is that the funds with longer measurement periods will have higher long-run Sharpe ratios than the funds with shorter measurement periods.

\footnotetext{
${ }^{9}$ As shown in the appendix, Sharpe ratio is equal to $\sqrt{\Lambda(0, T)-1}$ also with a stochastic price of risk process $\theta$.
} 
We now analyze the optimal trading strategies. The following result is an easy consequence of expression (28) in the appendix.

Proposition 3 The optimal trading strategy of the long-run manager is given by,

$$
\pi_{L}(t)=x\left[\left(e^{b \mathcal{T}}-\frac{\left(e^{b \mathcal{T}}-e^{r \mathcal{T}}\right)}{\Lambda(0, \mathcal{T})-1}\right) e^{-r(\mathcal{T}-t)}-\frac{X_{L}(t)}{x}\right]\left(\sigma^{\top}(t)\right)^{-1} D(t),
$$

where $D(t)$ is a process which is independent of preferences and which is defined in the appendix. Similarly, let $X_{S}\left(T_{n}\right)$ be the value of the fund managed by the short-run manager at time $T_{n}$ as the result of the minimization in (8). Then, for $n=0, \ldots, N-1, t \in\left[T_{n}, T_{n+1}\right)$

$$
\pi_{S}(t)=X_{S}\left(T_{n}\right)\left[\left(e^{b \tau}-\frac{\left(e^{b \tau}-e^{r \tau}\right)}{\Lambda\left(T_{n}, T_{n+1}\right)-1}\right) e^{-r\left(T_{n+1}-t\right)}-\frac{X_{S}(t)}{X_{S}\left(T_{n}\right)}\right]\left(\sigma^{\top}(t)\right)^{-1} D_{n}(t),
$$

where $D_{n}(t)$ is a preference free process given in the appendix.

Proposition 3 formalizes how the the Sharpe ratio maximizing trading strategy depends on the past performance. As seen in equations (10) and (11), the optimal trading strategy depends on $X(t)$ (even when divided by $X(t)$, unlike the optimal CRRA strategies) and hence it depends indirectly also on the past history of the stocks returns.

Note that in this section we have not made any specific assumptions about the functional form of $\sigma(t)$ and $\mu(t)$. In particular, they both can be history dependent. In the following sections, we will make more specific assumptions about $\mu(t)$ and $\sigma(t)$ in order to study quantitatively the potential impact of the horizon problem on Sharpe ratios as well as the implications of the investor's/managers stocks holdings.

\section{Horizon Problem in the IID Environment}

In this section, we study, as a benchmark, the simple case where the investment opportunity set is constant (the constant coefficients model). This continuous-time model corresponds exactly to the binomial model in Section 2. It illustrates further, in the continuous time setting, the basic observation from Section 2, how the two Sharpe ratios, $\mathcal{S}_{L}$ and $\mathcal{S}_{S}$ differ. It will also provide results on how significant the difference is quantitatively. The following proposition expresses the optimal strategy in terms of current wealth. 
Proposition 4 Assume $\mu(t)=\mu$ is a constant vector and $\sigma(t)=\sigma$ is a constant invertible matrix. The optimal strategy of the long-run manager is given by,

$$
\pi_{L}(t)=x\left(\frac{e^{\left(b-r+\theta^{2}\right) \mathcal{T}}-1}{e^{\theta^{2} \mathcal{T}}-1} e^{r t}-\frac{X_{L}(t)}{x}\right)\left(\sigma^{\top}\right)^{-1} \theta
$$

The trading strategy used by the short-run manager is given by, for $t \in\left[T_{n}, T_{n+1}\right), n=0$, $\ldots, N-1$,

$$
\pi_{S}(t)=X_{S}\left(T_{n}\right)\left(\frac{e^{\left(b-r+\theta^{2}\right) \tau}-1}{e^{\theta^{2} \tau}-1} e^{r\left(t-T_{n}\right)}-\frac{X_{S}(t)}{X_{S}\left(T_{n}\right)}\right)\left(\sigma^{\top}\right)^{-1} \theta .
$$

The time $t$ conditional expected return and conditional variance of the strategy initiated at $t=0$ by the long-run manager with horizon $\mathcal{T}$ are given by

$$
E\left[\frac{X_{L}(\mathcal{T})}{X_{L}(t)} \mid \mathcal{F}_{t}\right]=e^{r(\mathcal{T}-t)} e^{-\theta^{2}(\mathcal{T}-t)}+\frac{x}{X_{L}(t)}\left(e^{b \mathcal{T}}+\frac{e^{b \mathcal{T}}-e^{r \mathcal{T}}}{e^{\theta^{2} \mathcal{T}}-1}\right)\left(1-e^{-\theta^{2}(\mathcal{T}-t)}\right)
$$

and

$$
\operatorname{var}\left[\frac{X_{L}(\mathcal{T})}{X_{L}(t)} \mid \mathcal{F}_{t}\right]=e^{-\theta^{2}(\mathcal{T}-t)}\left(1-e^{-\theta^{2}(\mathcal{T}-t)}\right)\left[\frac{x}{X_{L}(t)}\left(e^{b \mathcal{T}}+\frac{e^{b \mathcal{T}}-e^{r \mathcal{T}}}{e^{\theta^{2} \mathcal{T}}-1}\right)-e^{r(\mathcal{T}-t)}\right]^{2}
$$

where $\theta=\sigma^{-1}(\mu-r)$ and $\theta^{2}$ is defined as $\theta^{\top} \theta$.

Proposition 4 shows that the Sharpe ratio maximizing dynamic portfolio strategy in an iid return environment has the following property: the amount invested in the risky assets decreases after good performance and vise versa. From the equations (14)-(15), we see that an implication of this property is that when past returns are low (high), the targeted expected return is high (low) and the associated variance is high (low). This is a more general formulation of the intuition illustrated in the example in Section 2.

The following proposition is obtained by straightforward computations.

Proposition 5 If $\mu(t)=\mu$ is a constant vector and $\sigma(t)=\sigma$ is a constant invertible matrix, then

$$
\mathcal{S}_{L}=\sqrt{e^{\theta^{2} \mathcal{T}}-1}
$$

and

$$
\mathcal{S}_{S}=\frac{e^{b \mathcal{T}}-e^{r \mathcal{T}}}{\sqrt{\left[e^{2 b \tau}+\frac{\left(e^{b \tau}-e^{r \tau}\right)^{2}}{e^{\theta^{2} \tau}-1}\right]^{N}-e^{2 b \mathcal{T}}}}
$$

where $\theta=\sigma^{-1}(\mu-r)$ and $\theta^{2}$ is defined as $\theta^{\top} \theta$. In particular, $\mathcal{S}_{L}>\mathcal{S}_{S}$ for $N>1$. 
It is worthwhile to note that for a fixed horizon, when the short-term horizon becomes arbitrarily small (i.e, when $N=\infty$ and $\mathcal{T}<\infty$ ), the optimal Sharpe ratio of the short-term manager converges to a constant,

$$
\lim _{N \uparrow \infty} \mathcal{S}_{S}=\frac{1-e^{-(b-r) \mathcal{T}}}{\sqrt{e^{\frac{(b-r)^{2}}{\theta^{2}} \mathcal{T}}-1}} .
$$

In particular, in the limit, the manager's trading strategy maximizes the instantaneous Sharpe ratio. In this case, the manager's investment behavior is in fact consistent with that of a particular CRRA utility maximizer. ${ }^{10}$ Moreover, since, for a given time horizon, the optimal Sharpe ratio does not depend on the expected return $b$, as is seen from (16), the manager who wants to maximize the instantaneous Sharpe ratio can use the optimal portfolio of any CRRA utility maximizer (Merton (1971)).

In order to get a sense of how significant the difference $\mathcal{S}_{L}-\mathcal{S}_{S}$ can be quantitatively, we calibrate the model to a set of parameters broadly consistent with the market data under the iid assumption. We take $r=3 \%$. We assume that there is a single stock with the volatility $\sigma=20 \%$ and the mean $\mu=10 \%$, resulting in $\theta=0.35$. We set $\tau$ to be a quarter, in agreement with quarterly return reports. Using such a short period will highlight the quantitative importance of the potential difference in Sharpe ratios due to the different investment horizons. We perform the calibration for three levels of $b: 6 \%, 10 \%$ and $14 \%$. The results are reported in Table 1 . The three panels in Table 1 correspond to the three levels of $b$. The main message of the table can be seen across columns within each panel. We see that $\mathcal{S}_{L}$ increases as the investment horizon increases, which is not always true for $\mathcal{S}_{S}$. For example, in panels two and three, $\mathcal{S}_{S}$ first rises and then falls. Quantitatively, the difference between $\mathcal{S}_{L}$ and $\mathcal{S}_{S}$ is alarmingly large. Even with the one year investment horizon of the investor, the difference ranges from $3.7 \%$ to $8.5 \%$ across the three panels. With a longer investment horizon, say 5 years, the difference can be as big as $68 \%$. Since both the trading strategy followed by the long-run manager and the strategy used by the short-run manager

\footnotetext{
${ }^{10}$ The intuition behind this result is that when the managers measurement period is arbitrarily small, they are facing a stationary problem. Consequently, they use the same portfolio proportion over time, consistent with power utility maximization. To establish the result mathematically, one must compute the limit of (13) when the interval $\left[T_{n}, T_{n+1}\right]$ becomes small. In the single asset case this limit will give

$$
\frac{\pi_{S}(t)}{X(t)}=\frac{b-r}{\theta^{2}} \frac{1}{\sigma} \theta
$$

This is the same as the optimal strategy for an agent with power utility $u(c)=\frac{c^{1-\gamma}}{1-\gamma}$ where $\gamma=\left(\frac{b-r}{\theta^{2}}\right)^{-1}$. In fact, there is a one-to-one mapping between the set of optimal portfolios for a power utility maximizers (indexed by the relative risk aversion parameter $\gamma$ ) and the set of the "instanteneous" Sharpe ratio maximizers (indexed by the targeted expected return $b$ ).
} 
produce the same expected return $b$, the difference in $\mathcal{S}_{L}$ and $\mathcal{S}_{S}$ corresponds exactly to that in the risk of the two portfolios. For example, for a one-year horizon, the risk of the portfolio managed by the short-run manager is $3.7 \%$ higher than that of the long-run portfolio, when $b=6 \%$.

\section{$5 \quad$ Horizon Problem in a Mean-Reverting Environment}

In the previous section, we studied the Sharpe ratio maximizing portfolio strategies as well as the associated horizon problem in the context of an iid investment opportunity set. In this section, we assume a more realistic investment environment, one in which the expected stock returns follow a mean-reverting process. There is a significant literature that shows that in a mean-reverting environment, investors with a short investment horizon exhibit a "trend chasing" behavior while those with a long investment horizon tend to hold more stocks (Barberis (2000), Watcher (2002)). By trend chasing or "momentum" strategies, we mean buying stocks that have been performing well, and selling stocks that have had low returns. In this section, we study whether such behavior may be consistent with maximizing Sharpe ratios, and whether the changing investment opportunity set has a quantitative effect on the difference of the Sharpe ratios of the long-run manager and the short-run manager.

In the previous section, Sharpe ratios $\mathcal{S}_{L}$ and $\mathcal{S}_{S}$ depend only on $b, r$ and $\theta^{2}$. The fact that there are more than one risky assets is not crucial. This motivates us to make a simplifying assumption in this section that there is only one stock (perhaps an index). We consider a model similar to Wachter (2002). The stock price process is given by,

$$
\frac{d S(t)}{S(t)}=(r+\sigma \theta(t)) d t+\sigma d W(t)
$$

where $\theta(t)$ is the price of risk, $r$ and $\sigma$ are riskless rate and volatility of the stock return, respectively. We assume that both $r$ and $\sigma$ are constant and that the price of risk $\theta(t)$ follows a mean-reverting process.

$$
d \theta(t)=-\lambda(\theta(t)-\bar{\theta}) d t-\sigma_{\theta} d W(t), \quad \theta(0)>0,
$$

where $\lambda, \bar{\theta}, \sigma_{\theta}$ are given fixed parameters. In particular, the stock price process is negatively correlated with the price of risk process. For technical reasons, we assume $\lambda>(2+\sqrt{2}) \sigma_{\theta}$. The basic reason behind this assumption is that if $\lambda$ is too small relative to $\sigma_{\theta}$, the force for mean-reversion would be too weak and the movement of $\theta(t)$ would be mainly determined by the random shocks coming from $\sigma_{\theta} d W(t)$. 
Proposition 6 The trading strategy of the long-run manager is given by

$\pi_{L}(t)=\frac{x}{\sigma}\left[\left(e^{(b-r) \mathcal{T}}+\frac{\left(e^{(b-r) \mathcal{T}}-1\right)}{\Lambda(\mathcal{T}, \theta(0))-1}\right) e^{r t}-\frac{X_{L}(t)}{x}\right]\left(\left(1+A(\mathcal{T}-t) \sigma_{\theta}\right) \theta(t)+B(\mathcal{T}-t) \sigma_{\theta}\right)$,

where $e^{11}$

$$
\Lambda(\mathcal{T}, \theta)=\exp \left[A(\mathcal{T}) \frac{\theta^{2}}{2}+B(\mathcal{T}) \theta+C(\mathcal{T})\right]
$$

and the expressions for $A(t), B(t)$ and $C(t)$ can be found in the appendix. The Sharpe ratio is given by

$$
\mathcal{S}_{L}=\sqrt{\Lambda(\mathcal{T}, \theta(0))-1}
$$

It is interesting to contrast the optimal stock holdings in the mean-reverting case with that in the iid case given in equation (12). We know from the previous section that in the iid case, the investor's strategy has a reversal feature. That is, when the portfolio has done well, other things being equal, the holdings of the risky asset tend to be reduced, and vice-versa. In the mean reverting case, we cannot say that the long-run manager always follows the reversal strategy ${ }^{12}$. In fact, there is an additional force, the price of risk $(\theta)$, that is at work in the mean reverting context. The equation (20) says that when $\theta(t)$ is high, the holding of the stock is high (the term in the bracket on the right-hand side of expression (20) is always positive and $A(t)>0$ as shown in the appendix). The intuition is that, because $\theta(t)$ is mean-reverting, when $\theta(t)$ is high, it is likely to fall in the future, as is the expected return on the stock. Then the long-run manager should hold more of the stock today, other things being equal. However, despite this additional force, the investor's strategy with mean reverting returns does have the reversal feature when the model is calibrated along the lines of Wachter (2002) .

For the short-run manager, we have

Proposition 7 The trading strategy of the short-run manager is given by, for $t \in\left[T_{n}, T_{n+1}\right)$, $n=0, \ldots, N-1$,

$$
\begin{aligned}
\pi_{S}(t)= & \frac{X_{S}\left(T_{n}\right)}{\sigma}\left[\left(e^{(b-r) \tau}+\frac{\left(e^{(b-r) \tau}-1\right)}{\Lambda\left(\tau, \theta\left(T_{n}\right)\right)-1}\right) e^{r\left(t-T_{n}\right)}-\frac{X_{S}(t)}{X_{S}\left(T_{n}\right)}\right] \\
& \times\left(\left(1+A\left(T_{n+1}-t\right) \sigma_{\theta}\right) \theta(t)+B\left(T_{n+1}-t\right) \sigma_{\theta}\right) .
\end{aligned}
$$

\footnotetext{
${ }^{11}$ Here, for simplicity, we abuse the notation a bit by writing $\Lambda(t, T)=\Lambda(T-t, \theta(t))$. This is justified because parameters in (18) and (19) are time invariant, and $\theta(t)$ is the only state variable.

${ }^{12}$ Our numerical computations indicate that when the risk premia is negative and the investment horizon is small, it may be optimal for the investor to adopt a momentum strategy. That is, if the risky asset has performed well, increase the investment therein, and vice-versa.
} 
The conditional instantaneous variance of the optimally managed portfolio is

$$
\begin{aligned}
& \frac{1}{\sigma^{2}}\left[\frac{X_{S}\left(T_{n}\right)}{X_{S}(t)}\left(e^{(b-r) \tau}+\frac{\left(e^{(b-r) \tau}-1\right)}{\Lambda\left(\tau, \theta\left(T_{n}\right)\right)-1}\right) e^{r\left(t-T_{n}\right)}-1\right]^{2} \\
& \times\left(\left(1+A\left(T_{n+1}-t\right) \sigma_{\theta}\right) \theta(t)+B\left(T_{n+1}-t\right) \sigma_{\theta}\right)^{2}
\end{aligned}
$$

To assess quantitatively the importance of a changing investment environment relative to the iid environment studied in Section 4, we calibrate the model parameters to those in Watcher (2002). The results are reported in the first three panels of Table 2. It is seen that, while $S_{L} / S_{S}-1$ depends on the initial $\theta_{0}$, it is always significantly different from zero. For example, for $\theta_{0}=0.1$ and with a one-year horizon, $S_{L} / S_{S}-1=1.0574$.

We mentioned above that in a mean-reverting environment the difference in Sharpe ratios is expected to be larger than in an iid environment. Indeed, in all cases (the first three panels), the values of $S_{L} / S_{S}-1$ are significantly larger than those reported in the first panel of Table $1 .^{13}$ It should be noted, however, that the cases studied in the first four panels in Table 2 are not directly comparable to the iid environment because in the iid environment the expected returns of the risky assets are constant while in the mean-reverting environment the expected returns are time-varying. To make the comparison as close as possible, we considered an iid environment where the expected return of the risky asset is equal to the long-run expected return of (18). That is, we set $\theta(t)$ to its long-run average $\bar{\theta}$ and set $\sigma_{\theta}=0$. We then considered a mean-reverting environment with $\theta_{0}=\bar{\theta}$. Thus, at time zero, in these two environments the stock has the same expected return. As time progresses, the expected returns start to deviate, but they still have the same long-run average. The result is reported in the last two panels of Table 2. Again, the Sharpe ratios differ. For example, at one year horizon, $S_{L} / S_{S}-1=0.0753$ in the iid environment, but $S_{L} / S_{S}-1=1.2659$ in the mean-reverting environment. Moreover, as the horizon becomes longer, the difference becomes bigger. For example, the ratio of $S_{L} / S_{S}-1$ for the 5 -year horizon to that for the one-year horizon in the iid environment is $0.6328 / 0.0753=8.4$, while that in the meanreverting environment is $42.6018 / 1.2659=33.7$. Intuitively, this is due to the fact that the long-run risk in a mean-reverting environment is lower.

It is also worth noting that for both the long-run and the short-run managers, ignoring the predictability (the mean-reversion) will lead to an economically significant error in the Sharpe ratio measurement especially for long horizons. For instance, for a five-year horizon, ignoring the mean reversion will underestimate the investor's Sharpe ratio. The intuition behind this result is the following: since returns are mean reverting, the cumulative variance

\footnotetext{
${ }^{13}$ In the calculation of Table 2, we have assumed that $b=6 \%$.
} 
of the return shocks at long horizons grows less than linearly with the horizon $T$. On the other hand, when the returns are iid, the cumulative returns variance grows linearly with $T$ and consequently the Sharpe ratio is lower than in the mean reverting case.

\section{Time Series Properties of the Optimal Strategy}

We consider again the general model (2). The following proposition is a corollary of Proposition 3 that focuses on the risk of the trading strategy. We have already obtained analogous results for the special models of the previous sections.

Proposition 8 At any intermediate time $t \in(0, T)$, the Sharpe ratio maximizing strategy initiated at time $t=0$ by the manager with horizon $\mathcal{T}$ generates the conditional expected return

$$
E\left[\frac{X_{L}(\mathcal{T})}{X_{L}(t)} \mid \mathcal{F}_{t}\right]=\frac{e^{r(\mathcal{T}-t)}}{\Lambda(t, \mathcal{T})}+\frac{x}{X_{L}(t)}\left(e^{b \mathcal{T}}+\frac{e^{b \mathcal{T}}-e^{r \mathcal{T}}}{\Lambda(0, \mathcal{T})-1}\right)\left(1-\frac{1}{\Lambda(t, \mathcal{T})}\right)
$$

and the conditional variance

$$
\operatorname{var}\left[\frac{X_{L}(\mathcal{T})}{X_{L}(t)} \mid \mathcal{F}_{t}\right]=\frac{1}{\Lambda(t, \mathcal{T})}\left(1-\frac{1}{\Lambda(t, \mathcal{T})}\right)\left[\frac{x}{X_{L}(t)}\left(e^{b \mathcal{T}}+\frac{e^{b \mathcal{T}}-e^{r \mathcal{T}}}{\Lambda(0, \mathcal{T})-1}\right)-e^{r(\mathcal{T}-t)}\right]^{2}
$$

This proposition shows that the risk of the Sharpe ratio maximizing portfolio over the interval $[t, \mathcal{T}]$ depends on $X_{L}(t) / x$. If the portfolio performed well in the past so that $X_{L}(t) / x$ is large, then the manager will lower the risk of the portfolio over $[t, \mathcal{T}] .{ }^{14}$ This type of behavior is sometimes labeled "risk manipulation" in the performance literature, e.g. Brown et al (1996), Chevalier and Ellison (1997). The explanation offered by those papers for such behavior is largely based on the tournament theory, that is, on the fact that funds compete for a limited pool of investment money. Here, we have shown that similar behavior is also implied by a standard portfolio choice model, as the trading strategy which maximizes Sharpe ratio exhibits the "risk manipulation" properties. Note that the proposition is established without any specific assumptions on the return dynamics, and thus the "risk manipulation" feature appears to be a fundamental implication of the Sharpe ratio maximization.

In order to get a sense of the prediction's magnitude in our model, we did a simple simulation exercise. Using a dataset of monthly returns of growth-oriented mutual funds

\footnotetext{
${ }^{14}$ It can be shown using the results in the appendix that the expression in the square bracket in equation (24) is always positive, and we also have, by Jensen's inequality, $\Lambda(t, \mathcal{T})>1$.
} 
between 1980 and 1991, Brown et al (1996) documented that the managers risk behavior depends on the realized performance. More specifically, one of the exercises that Brown et al (1996) performed is to test if the fund's relative performance in the first six months of the year explains the change of the fund's volatility in the second part of the year. For that purpose, they construct two variables. First, for each year during the sample period, the 6 -months cumulative return variable for each fund $j$ is defined by

$$
R_{j}=\left(1+r_{1, j}\right)\left(1+r_{2, j}\right) \ldots\left(1+r_{6, j}\right)-1
$$

In the above notation, $r_{i, j}$ represents the rate of return of the fund $j$ during the month $i$. For each year, the sample of funds is divided into two subsets, the "losers" and the "winners" according to the ranking of the fund with respect to the variable $R$ (see the explanations in Table 3). Second, Brown et al (1996) constructed the "risk adjustment ratio" variable $R A R$ to assess the risk behavior of each fund. For each year, the variable $R A R$ for the the fund $j$ is defined by

$$
R A R_{j}=\frac{\sqrt{\frac{\sum_{i=7}^{12}\left(r_{i}-\bar{r}_{7,12}\right)^{2}}{5}}}{\sqrt{\frac{\sum_{i=1}^{6}\left(r_{i}-\bar{r}_{1,6}\right)^{2}}{5}}}
$$

where $\bar{r}_{1,6}$ (resp. $\bar{r}_{7,12}$ ) represent the rate of return over the first (resp. last) six months of a given year. For each year, each sub-sample (formed on the basis of "R") is in turn divided into two sub-samples, "high RAR" and "low RAR", where high and low are defined relative to the median RAR in the cross section of funds within the same year.

Brown et al (1996) construct a $2 \times 2$ contingency table according to the variable $R$ ("losers/winners") and the variable $R A R$. They show that the ratio $R A R$ is larger for the loser funds subset than for the winner funds subset. In the base case scenario, their findings are reported in Table 3 in the line labeled BHS. Our exercise simply consists of reproducing the $2 \times 2$ contingency table in the context of a simulated sample of 3000 fictitious funds ${ }^{15}$ which maximize the one year Sharpe ratio with expected returns $b=8.3 \%, b=9 \%, b=13 \%$, $b=16 \%, b=20 \%$ and $b=25 \%$. We assume that the fund manager uses one risky asset, called index, and a risk-free asset, and that the index returns are iid. We calibrate the parameters $(\mu, \sigma, r)$ with the 3 months US treasury bills and an US value-weighted index. We use monthly returns from the Kenneth French's web site ${ }^{16}$. The results of the simulation

\footnotetext{
${ }^{15}$ The size of the sample roughly matches the Brown et al (1996) size which is around 334 funds for 12 years.

${ }^{16}$ http://mba.tuck.dartmouth.edu/pages/faculty/ken.french/
} 
exercise are systematically ${ }^{17}$ consistent with the theoretical predictions of Proposition 8 that is, the ratio RAR is lower for the "winner" funds. The effect is actually higher than the empirical results, possibly due to the choice of the iid model and the specific calibration we employ, or because the Sharpe ratio maximization is only one factor in the investment strategy of the considered funds. Nevertheless, the simulation results seem to provide a correct order of magnitude for the frequency of the funds exhibiting the "risk manipulation" behavior.

\section{Conclusion}

In this paper, we study a multi-period framework in which a portfolio manager with a shortterm horizon maximizes, over a long horizon, the Sharpe ratio of the managed portfolio. Two implications we found should be of interest to the literature on fund management. The first implication is that the strategy of maximizing the short-term Sharpe ratio can result in a significant loss of performance for investors who care about the long-term Sharpe ratio. The second implication is that the trading strategy that maximizes Sharpe ratio exhibits the risk manipulation features documented in the literature (e.g., Brown et al (1996), and Chevalier and Ellison (1997)) within the measurement period. That is, the funds that perform poorly in the first half of the performance measurement period tend to increase their risk in the second half, and vice-versa.

This second implication suggests that the "risk manipulation" behavior documented in the literature is not necessarily due to the funds' managers caring about attracting new investments in the following year, while competing with other funds for those investments. In fact, the observed behavior is consistent with the prediction of our more standard portfolio choice model, in which the manager maximizes the short-term Sharpe ratio. The existing empirical studies of the risk manipulation behavior are mostly based on the former, socalled tournament view of investment, but our findings show that such behavior may have alternative explanations. It would be of considerable interest to conduct further empirical studies to test which, if either, of the two theoretical explanations is more valid.

Finally, an important practical question is whether there are simple and natural performance measures other than the Sharpe ratio, that could be used for managers' compensation, and which would make the interests of the managers and the investors of differing horizons

\footnotetext{
${ }^{17}$ We tried various sample sizes $(3000,5000,10000)$ as well as other parameters for $(\mu, \sigma, r)$. The results always have the same direction and we can fit closer the BHS empirical findings if we give ourselves the freedom to pick those parameters.
} 
more closely aligned. At the broader level, it would be interesting to study the welfare implications of the Sharpe ratio performance measure, for example in the terms of expected utility. For instance, a long-run investor with a CRRA utility may be better off investing with a short-run than with a long-run Sharpe ratio maximizer, depending on the model and the parameters. We leave this task for future research. 


\section{A Appendix}

Proof of Proposition 1: We provide a detailed proof for completeness, although there are proofs available in the literature mentioned at the end of Introduction.

First let us define for each $\lambda>0$ and $d>0$ an unconstrained version of the problem (6),

$$
\inf _{\pi \in \mathcal{A}(x)} E\left[(X(T)-d)^{2}+\lambda(X(T)-d)\right]
$$

which we will solve by duality. Define the convex function

$$
R(x)=(x-d)^{2}+\lambda(x-d)
$$

and its dual function $\tilde{R}$ defined for each $y \in \mathbb{R}$ by

$$
\tilde{R}(y) \equiv \inf _{x}(R(x)-y x)=R\left(d+\frac{1}{2}(y-\lambda)\right)-y\left(d+\frac{1}{2}(y-\lambda)\right) .
$$

Now, for all $x, y \in \mathbb{R}$ and $\pi \in \mathcal{A}(x)$,

$$
R(X(T)) \geq \tilde{R}(y \xi(T))+y \xi(T) X(T) .
$$

In particular, if we take expectations in (26) we get for all $x, y \in \mathbb{R}$ and $\pi \in \mathcal{A}(x)$, by definition of $\mathcal{A}(x)$,

$$
E[R(X(T))] \geq E[\tilde{R}(y \xi(T))+y x] .
$$

This gives a lower bound for $E[R(X(T))]$, which is attained if we choose a portfolio $\hat{\pi}$ so that the associated terminal wealth is

$$
\hat{X}(T)=d+\frac{1}{2}(y \xi(T)-\lambda) .
$$

Such a portfolio exists if we choose $y$ so that $E[\xi(T) \hat{X}(T)]=x$ (see Karatzas and Shreve (1998)). This is satisfied if

$$
y=2(x-E(\xi(T)(d-\lambda / 2))) / E\left(\xi^{2}(T)\right) .
$$

To recap, the optimal terminal wealth of the unconstrained problem $(25)$ is $\hat{X}(T)$ and the value function is given by

$$
V(\lambda, x, d)=\frac{(x-E(\xi(T)(d-\lambda / 2)))^{2}}{E\left(\xi^{2}(T)\right)}-\frac{\lambda^{2}}{4} .
$$


It can easily be verified that the objective function in the problem (6) is a convex function of $\pi$. Additionally, the constraint in the problem (6) is linear and therefore the subset of $\mathcal{A}(x)$ that satisfies this constraint is convex. Therefore, from Luenberger (1979), we know that the value function of the constrained problem (6) is

$$
V(x)=\max _{\lambda} V\left(\lambda, x, x e^{b T}\right)
$$

and one can easily check that the optimum is attained by

$$
\lambda^{*}=2 x \frac{E(\xi(T))}{\operatorname{Var}(\xi(T))}\left(1-e^{b T} E(\xi(T))\right) .
$$

After a substitution in equation (27) we have

$$
y^{*}=2 x \frac{1}{\operatorname{Var}(\xi(T))}\left(1-e^{b T} E(\xi(T))\right) .
$$

Furthermore, from Luenberger (1979) the optimal terminal wealth of the unconstrained problem (6) is

$$
X^{*}(T)=x e^{b T}-x \frac{e^{b T} E(\xi(T))-1}{\operatorname{Var}(\xi(T))}(\xi(T)-E(\xi(T)))
$$

Now, it is easy to check that

$$
\operatorname{Var}\left(X^{*}(T)\right)=x^{2} \frac{E^{2}(\xi(T))}{\operatorname{Var}(\xi(T))}\left(e^{b T}-\frac{1}{E(\xi(T)}\right)^{2},
$$

and therefore the efficient frontier in the portfolio diagram is a straight line defined by

$$
\sigma=x \frac{E(\xi(T))}{\sqrt{\operatorname{Var}(\xi(T))}}\left|e^{b T}-\frac{1}{E(\xi(T))}\right| .
$$

Thus, the Sharpe ratio of the optimal portfolio is given by

$$
\mathcal{S} \equiv \frac{\left|E\left(X^{*}(T)\right)-\frac{x}{E(\xi(T))}\right|}{\sqrt{\operatorname{Var}\left(X^{*}(T)\right)}}=\frac{\sqrt{\operatorname{Var}(\xi(T))}}{E(\xi(T))}=\frac{\sqrt{\operatorname{Var}(Z(T))}}{E(Z(T))}=\sqrt{\operatorname{Var}(Z(T))}=\sqrt{\Lambda(0, T)-1} .
$$

Note finally, that the results of the current section are quite general and do not necessitate any assumptions on the investment opportunity set other than market completeness.

Proof of Corollary 2: We denote by $X_{S}^{b}$ the wealth for the short-run manager when the expected return is $b>r$ and $X_{L}^{b}$ the wealth for the long-run manager when the expected return is $b>r .{ }^{18}$ By taking the successive conditional expectations of the constraint (9)

$$
E\left[X_{S}^{b}(\mathcal{T}) \mid \mathcal{F}_{T_{N-1}}\right]=X_{S}^{b}\left(T_{N-1}\right) e^{b \tau}
$$

\footnotetext{
${ }^{18}$ This notation is only adopted for this proof.
} 
with respect to the sequence of the sigma algebras $\mathcal{F}_{T_{n}}, n=N-1, N-2, \ldots, 0$, one can get

$$
E\left[X_{S}^{b}(\mathcal{T})\right]=x e^{b \mathcal{T}}
$$

and hence the short-run manager's optimal strategy leads to a terminal wealth satisfying the long-run constraint $(7)$. Therefore, $\operatorname{var}\left(X_{S}^{b}(\mathcal{T})\right) \geq \operatorname{var}\left(X_{L}^{b}(\mathcal{T})\right)$. Consequently, Sharpe ratio of the long-run manager $\mathcal{S}_{L}^{b}$ is at least as high as Sharpe ratio of the short-run manager $\mathcal{S}_{S}^{b}$. However, Proposition 1 shows that the Sharpe ratio of the long-run manager is independent from the expected return and consequently, the inequality

$$
\mathcal{S}_{L} \geq \mathcal{S}_{S}
$$

holds for all $b>r$.

Proof of Proposition 3: Under our assumption of bounded $\theta$ the process

$$
N(t) \equiv E\left[Z(T)^{2} \mid \mathcal{F}_{t}\right]
$$

is a positive martingale and thus it must admit the representation

$$
N(t)=N_{0}+\int_{0}^{t} N(s) \phi^{\top}(s) d W(s)
$$

for a suitable adapted process $\phi$. On the other hand, $\Lambda(t, T)=N(t) / Z(t)^{2}$ and thus by Ito's Lemma

$$
d \Lambda(t, T)=[. .] d t+\Lambda(t, T)(\phi(t)+2 \theta(t))^{\top} d W(t)
$$

Now, under the assumption of constant interest rates, the wealth equation (28) translates into

$$
X^{*}(T)=x e^{b T}-x \frac{e^{b T}-e^{r T}}{\Lambda(0, T)-1}(Z(T)-1),
$$

and by the assumption of no arbitrage, the deflated wealth must be a martingale, that is,

$$
X^{*}(t)=E_{t}\left(\frac{\xi(T)}{\xi(t)} X^{*}(T)\right)=x e^{-r(T-t)}\left[e^{b T}-\frac{e^{b T}-e^{r T}}{\Lambda(0, T)-1}(\Lambda(t, T) Z(t)-1)\right] .
$$

Differentiating the above expression gives the martingale part of the wealth differential

$$
d X^{*}(t)=[. .] d t-x e^{-r(T-t)}\left[\frac{e^{b T}-e^{r T}}{\Lambda(0, T)-1}\right] Z(t) \Lambda(t, T)(\phi(t)+\theta(t))^{\top} d W(t) .
$$

Now identifying the above expression with the wealth dynamic (4), which translates to

$$
d X(t)=r X(t) d t+\pi^{\top}(t)\left[\sigma^{-1}(t) \theta(t) d t+\sigma(t) d W(t)\right]
$$


in vector notation, gives the first best optimal strategy in term of the processes $Z, \Lambda, \phi$ and $\theta$. In order to get the expression in equation (10), we just substitute $Z(t) \Lambda(t, T)$ by its expression in term of the optimal wealth from equation (30) and define the process $D$ as

$$
D(t) \equiv-\phi(t)-\theta(t)
$$

To get the manager strategy in equation (11), one just need to specialize the investor strategy with appropriate adjustments for the horizon and the wealth.

Proof of Proposition 8: To establish the expression (23), one must first compute the conditional expectation of (29) to obtain

$$
E\left[X_{L}(\mathcal{T}) \mid \mathcal{F}_{t}\right]=x e^{b \mathcal{T}}-x \frac{e^{b \mathcal{T}}-e^{r \mathcal{T}}}{\Lambda(0, \mathcal{T})-1}(Z(t)-1),
$$

Substituting, the current wealth given in the expression (30) (with $T=\mathcal{T}$ ) in the above expectation gives the expression (23). A similar method gives the return conditional variance (24). Notice that the conditional Sharpe ratio can be easily derived from (23) - (24) and it is given by

$$
\mathcal{S}_{t, \mathcal{T}}=\sqrt{\Lambda(t, \mathcal{T})-1}
$$

Proof of Proposition 5 : In the iid case, a Girsanov transformation gives the expression $\Lambda(0, \mathcal{T})=e^{\theta^{2} \mathcal{T}}$ and thus (16) is implied by Proposition 1 . To get (17), one need to substitute the expression of $\Lambda$ in (11) and get

$$
X_{S}(\mathcal{T})=x \prod_{n=0}^{N-1}\left[e^{b \tau}-\frac{\left(e^{b \tau}-e^{r \tau}\right)}{e^{\theta^{2} \tau}-1}\left(\frac{Z\left(T_{n+1}\right)}{Z\left(T_{n}\right)}-1\right)\right]
$$

In particular, noticing that $Z\left(T_{n+1}\right) / Z\left(T_{n}\right), n=0, \ldots, N-1$ are mutually independent one can get,

$$
\begin{aligned}
E\left(X_{S}(\mathcal{T})\right)^{2} & =x^{2} \prod_{n=0}^{N-1}\left[e^{2 b \tau}+\frac{\left(e^{b \tau}-e^{r \tau}\right)^{2}}{\left(e^{\theta^{2} \tau}-1\right)^{2}} E\left(\frac{Z\left(T_{n+1}\right)}{Z\left(T_{n}\right)}-1\right)^{2}\right] \\
& =x^{2}\left[e^{2 b \tau}+\frac{\left(e^{b \tau}-e^{r \tau}\right)^{2}}{e^{\theta^{2} \tau}-1}\right]^{N} .
\end{aligned}
$$

Thus,

$$
\operatorname{Var}\left(X_{S}(\mathcal{T})\right)=x^{2}\left[\left(e^{2 b \tau}+\frac{\left(e^{b \tau}-e^{r \tau}\right)^{2}}{e^{\theta^{2} \tau}-1}\right)^{N}-e^{2 b \mathcal{T}}\right]
$$


and (17) obtains.

Proof of Propositions 4: In the iid case, it can be calculate that

$$
\Lambda(t, T)=\exp \left(\theta^{2}(T-t)\right), \quad E\left[Z(T)^{2} \mid \mathcal{F}_{t}\right]=Z(t)^{2} \exp \left(\theta^{2}(T-t)\right)
$$

and thus $D(t)$ defined in the proof of Proposition 3 is equal to $\theta$.

Proof of Proposition 6 : Obviously, the mean reverting model fits in the class of models studied in the first section and to prove Proposition 6, we first need to compute the quantity

$$
\Lambda(T-t, \theta(t)) \equiv E\left[\left(\frac{Z(T)}{Z(t)}\right)^{2} \mid \mathcal{F}_{t}\right]
$$

in the context of this model and apply Proposition 1 to get the expression of the maximal Sharpe artio. Note that we use here the abuse of notation, $\Lambda(t, T, \theta(t))=\Lambda(T-t, \theta(t))$ legitimated by the fact the model parameters in (18) and (19) are time invariant. Then, to compute the first best optimal strategy, one need to compute the optimal wealth process and then identify its martingale part with the wealth dynamic (4). This part of the proof is very similar to the iid case (except that the Ito computations are more involved) and we omit it.

The following lemma provides the expression of $\Lambda$.

Lemma 9 : Under the assumption $\lambda^{* 2}-2 \sigma_{\theta}^{2}>0$, where we recall $\lambda^{*}=\lambda-2 \sigma_{\theta}$ the function $\Lambda$ has the closed form expression

$$
\Lambda(T-t, \theta(t))=\exp \left[A(T-t) \frac{\theta^{2}(t)}{2}+B(T-t) \theta(t)+C(T-t)\right]
$$

where

$$
\begin{aligned}
A(u)= & \frac{\lambda^{*}}{\sigma_{\theta}^{2}}+\frac{\beta}{\sigma_{\theta}^{2}} \frac{\alpha e^{-2 \beta u}+1}{\alpha e^{-2 \beta u}-1} \\
B(u)= & -\frac{\lambda^{*} \bar{\theta}^{*}}{\sigma_{\theta}^{2}}+\frac{2 \bar{\theta}^{*}}{\beta} \frac{(1+\alpha) e^{-\beta u}}{\alpha e^{-2 \beta u}-1}-\frac{2 \bar{\theta}^{*}}{\beta}\left(1+\frac{\beta^{2}}{2 \sigma_{\theta}^{2}}\right) \frac{\alpha e^{-2 \beta u}+1}{\alpha e^{-2 \beta u}-1} \\
C(u)= & \frac{\lambda^{*}}{2 \sigma_{\theta}^{2}} \bar{\theta}^{* 2}+\left(\frac{\lambda^{*}}{2}+\bar{\theta}^{* 2}-\frac{\beta}{2}+\frac{1}{2} \frac{\eta^{2}}{\beta^{2}}\right) u+\frac{\beta \bar{\theta}^{* 2}}{2 \sigma_{\theta}^{2}}\left(1+\frac{4 \sigma_{\theta}^{2}}{\beta^{2}}\right) \frac{\alpha e^{-2 \beta u}+1}{\alpha e^{-2 \beta u}-1} \\
& -2 \bar{\theta}^{* 2} \frac{(1+\alpha)}{\beta} \frac{e^{-\beta u}}{\alpha e^{-2 \beta u}-1}-\frac{1}{2} \ln \left[\frac{\alpha e^{-2 \beta u}-1}{\alpha-1}\right] \\
& +\frac{\eta^{2}}{2 \beta^{3}}\left(\frac{(\alpha+1)^{2}}{2 \alpha}+2\right)\left(\frac{1}{\alpha e^{-2 \beta u}-1}-\frac{1}{\alpha-1}\right) \\
& \quad-\eta^{2} \frac{\alpha+1}{\beta^{3}}\left(\frac{e^{-\beta u}}{\alpha e^{-2 \beta u}-1}-\frac{1}{\alpha-1}\right),
\end{aligned}
$$


and

$$
\beta \equiv \sqrt{\lambda^{* 2}-2 \sigma_{\theta}^{2}}, \quad \alpha \equiv \frac{\lambda^{*}-\beta}{\lambda^{*}+\beta}, \quad \eta \equiv 2 \sigma_{\theta} \bar{\theta}^{*}, \quad \bar{\theta}^{*}=\lambda \bar{\theta} /\left(\lambda-2 \sigma_{\theta}\right)
$$

Proof of Lemma 9 : First,let us state the following technical result:

Lemma 10 : Assume that $f$ and $g$ are two functions of class $C^{1}$ on $[0, T]$ and denote by $W^{y}$ a translated Brownian starting at $y \in(-\infty,+\infty)$ (i.e. $Y(t) \equiv y+W(t)$ ). Then we have the following property

$$
\begin{aligned}
E\{\exp & {\left[g(t) Y(t)+\frac{1}{2} f(t) Y^{2}(t)-\frac{1}{2} \int_{0}^{t}\left(f^{2}(s)+f^{\prime}(s)\right) Y^{2}(s) d s\right.} \\
& \left.\left.-\int_{0}^{t}\left(f(s) g(s)+g^{\prime}(s)\right) Y(s) d s\right]\right\} \\
=\exp & {\left[\frac{1}{2} f(0) y^{2}+g(0) y+\frac{1}{2} \int_{0}^{t} f(s) d s+\frac{1}{2} \int_{0}^{t} g^{2}(s) d s\right] }
\end{aligned}
$$

Proof of Lemma 10: Note that the process

$$
M(t) \equiv \exp \left[\int_{0}^{t}(f(s) Y(s)+g(s)) d Y(s)-\frac{1}{2} \int_{0}^{t}(f(s) Y(s)+g(s))^{2} d s\right],
$$

is a martingale. An integration by part gives

$$
\begin{aligned}
\int_{0}^{t}(f(s) Y(s)+g(s)) d Y(s)= & g(t) Y(t)-g(0) Y_{0}+\frac{1}{2}\left(f(t) Y^{2}(t)-f(0) Y_{0}^{2}\right) \\
& -\int_{0}^{t} g^{\prime}(s) Y(s) d s-\frac{1}{2} \int_{0}^{t}\left(f(s)+Y^{2}(s) f^{\prime}(s)\right) d s,
\end{aligned}
$$

and one can get (35) by substituting (36) in the equation $E(M(t))=1$.

Second, to pursue the proof of Lemma 9, one can easily get after a change of probability

$$
\Lambda(T-t, \theta(t))=E^{*}\left[\exp \left(\int_{t}^{T} \theta^{2}(s) d s\right) \mid \mathcal{F}_{t}\right]
$$

where $E^{*}$ is an expectation under a probability measure $P^{*}$ defined by

$$
\frac{d P^{*}}{d P}=\exp \left(-2 \int_{0}^{t} \theta(s) d W(s)-2 \int_{0}^{t}|\theta(s)|^{2} d s\right) .
$$

By Girsanov Theorem, the process $\theta$ follows the Ornstein Uhlenbeck dynamic

$$
d \theta(t)=-\lambda^{*}\left(\theta(t)-\bar{\theta}^{*}\right) d t-\sigma_{\theta} d W^{*}(t),
$$


where the process $W^{*}$ is defined by

$$
d W^{*}(t)=d W(t)+2 \theta(t) d t
$$

is a Brownian motion under $P^{*}$.

Let us now define another new probability $\bar{P}$ by

$$
\frac{d \bar{P}}{d P^{*}}=\exp \left(-\int_{0}^{t} \frac{\lambda^{*}}{\sigma_{\theta}}\left(\theta(s)-\bar{\theta}^{*}\right) d W^{*}(s)-\frac{1}{2} \int_{0}^{t} \frac{\lambda^{2 *}}{\sigma_{\theta}^{2}}\left|\theta(s)-\bar{\theta}^{*}\right|^{2} d s\right) .
$$

By Girsanov Theorem, the dynamic of $\theta$ under $\bar{P}$ is given by

$$
d \theta(t)=-\sigma_{\theta} d B(t)
$$

where

$$
B(t) \equiv W^{*}(t)+\frac{\lambda^{*}}{\sigma_{\theta}} \int_{0}^{t}(\theta(s)-\bar{\theta}) d s
$$

is a standard Brownian motion under the probability $\bar{P}$. An integration of (38) gives

$$
\frac{\theta(t)}{\sigma_{\theta}}=\frac{\theta(0)}{\sigma_{\theta}}-B(t)
$$

and proves that the process $\frac{\theta(t)}{\sigma_{\theta}}$ is a translated Brownian motion under the probability $\bar{P}$, starting at $\frac{\theta(0)}{\sigma_{\theta}}$. Thus letting

$$
v \equiv \frac{\theta(0)-\bar{\theta}}{\sigma_{\theta}}
$$

the process

$$
B_{v}(t) \equiv \frac{\theta(t)-\bar{\theta}}{\sigma_{\theta}}
$$

is also a translated Brownian motion under the probability $\bar{P}$, starting at $B_{v}(0)=v$.

Now, by Girsanov Theorem,

$$
\begin{aligned}
& \Lambda(T, \theta(0))=E^{*}\left[\exp \left(\int_{0}^{T} \theta^{2}(s) d s\right)\right]=\bar{E}\left[\frac{d P^{*}}{d \bar{P}} \exp \left(\int_{0}^{T} \theta^{2}(s) d s\right)\right] \\
= & \bar{E}\left[\exp \left(-\int_{0}^{T} \lambda^{*} B_{v}(s) d B_{v}(s)-\frac{1}{2} \int_{0}^{T} \lambda^{* 2} B_{v}^{2}(s) d s\right) \exp \left(\int_{0}^{T}\left(\bar{\theta}^{*}+\sigma_{\theta} B_{v}(s)\right)^{2} d s\right)\right],
\end{aligned}
$$

where $\bar{E}$ denotes the expectation under the probability measure $\bar{P}$.

Applying Ito's expansion to $B_{v}^{2}(s)$ allows to erase the stochastic integral in the above expression of $\Lambda$ and leads after some computations to

$$
\begin{aligned}
\Lambda(T, \theta(0))=\quad & \exp \left(\frac{\lambda^{*}}{2} v^{2}+\frac{\lambda^{*}}{2} T+\bar{\theta}^{* 2} T\right) \\
& \times \bar{E}\left[\exp \left(-\frac{1}{2} \int_{0}^{T}\left(\lambda^{* 2}-2 \sigma_{\theta}^{2}\right) B_{v}(s)^{2} d s+2 \int_{0}^{T} \sigma_{\theta} \bar{\theta}^{*} B_{v}(s) d s-\frac{\lambda^{*}}{2} B_{v}^{2}(T)\right)\right] .
\end{aligned}
$$


This last expression of $\Lambda$ puts us in position to apply Lemma 10 and reduce the problem of computing $\Lambda$ to an ordinary differential equation (ode) resolution problem. As a matter of fact, applying Lemma 10 under the probability $\bar{P}$ gives

$$
\begin{aligned}
\Lambda(T, \theta(0))= & \exp \left(\frac{\lambda}{2} v^{2}+\frac{\lambda}{2} T+\bar{\theta}^{2} T\right) \\
& \times \exp \left(\frac{1}{2} f(0) v^{2}+g(0) v+\frac{1}{2} \int_{0}^{T} f(s) d s+\frac{1}{2} \int_{0}^{T} g^{2}(s) d s\right)
\end{aligned}
$$

where the function $f$ solves the Riccati equation

$$
f^{2}(t)+f^{\prime}(t)=\lambda^{* 2}-2 \sigma_{\theta}^{2} ; \quad f(T)=-\lambda^{*},
$$

and where the function $g$ solves the linear ordinary differential equation

$$
f(t) g(t)+g^{\prime}(t)=-2 \sigma_{\theta} \bar{\theta}^{*} ; \quad g(T)=0 .
$$

Standard ode resolution techniques give, in case $\lambda^{* 2}-2 \sigma_{\theta}^{2}>0$,

$$
f(t)=\beta \frac{\alpha e^{-2 \beta(T-t)}+1}{\alpha e^{-2 \beta(T-t)}-1},
$$

and

$$
g(t)=\frac{\eta}{\beta} \frac{(\alpha+1) e^{-\beta(T-t)}-\left(1+\alpha e^{-2 \beta(T-t)}\right)}{\alpha e^{-2 \beta(T-t)}-1} .
$$

Now incorporating (39) in the expression (40) gives

$$
\begin{array}{r}
\Lambda(T, \theta(0))=\exp \left[\frac{1}{2} \frac{f(0)+\lambda^{*}}{\sigma_{\theta}^{2}} \theta^{2}(0)-\left(\frac{f(0)+\lambda^{*}}{\sigma_{\theta}^{2}} \bar{\theta}^{*}-\frac{g(0)}{\sigma_{\theta}}\right) \theta(0)\right] \\
\times \exp \left[\frac{1}{2} \frac{f(0)+\lambda^{*}}{\sigma_{\theta}^{2}} \bar{\theta}^{* 2}-\frac{g(0)}{\sigma_{\theta}} \bar{\theta}^{*}+\left(\bar{\theta}^{* 2}+\frac{\lambda^{*}}{2}\right) T+\frac{1}{2} \int_{0}^{T} f(s) d s+\frac{1}{2} \int_{0}^{T} g^{2}(s) d s\right]
\end{array}
$$

and identifying the last expression of $\Lambda$ with (34) gives immediately the expressions of $A$ and $B$ provided in Lemma 9. An additional integration exercise must however be done to get the expression of $C$.

After some integration by parts, it can be proven that

$$
\frac{1}{2} \int_{0}^{T} f(t) d t=-\frac{\beta}{2} T-\frac{1}{2} \ln \left[\frac{\alpha e^{-2 \beta T}-1}{\alpha-1}\right],
$$

and, for $T$ small enough,

$$
\begin{aligned}
& \int_{0}^{T} \frac{1}{2} g^{2}(t) d t=\frac{T}{2} \frac{\eta^{2}}{\beta^{2}}+\frac{\eta^{2}}{2 \beta^{3}}\left(\frac{(\alpha+1)^{2}}{2 \alpha}+2\right)\left(\frac{1}{\alpha e^{-2 \beta T}-1}-\frac{1}{\alpha-1}\right) \\
& -\eta^{2} \frac{\alpha+1}{\beta^{3}}\left(\frac{e^{-\beta T}}{\alpha e^{-2 \beta T}-1}-\frac{1}{\alpha-1}\right) .
\end{aligned}
$$

One can now easily conclude the expression of $C$ by incorporating the expression $\int_{0}^{T} f(t) d t$ and $\int_{0}^{T} g^{2}(t) d t$ into equation (41). 


\section{Table 1: Sharpe Ratios for various levels of $b$ and investment horizons}

This table displays the two Sharpe ratios $\mathcal{S}_{L}$ and $\mathcal{S}_{S}$ (for a quarterly frequency) as the investment horizon and the expected return $b$ vary. In order to judge whether the difference in investment horizon has significant effect on the Sharpe ratios, the table also reports the ratio of the two Sharpe ratios. In the calibration, we have assumed that $r=3 \%, \sigma=20 \%$ and $\mu=10 \%$. We look at three levels of $b=6 \%, 10 \%$ and $14 \%$.

\begin{tabular}{|c|c|c|c|c|c|c|c|}
\hline year & $1 / 2$ & 1 & 2 & 5 & 10 & 20 & 30 \\
\hline \hline \multicolumn{8}{|c|}{ Panel with $b=6 \%$} \\
\begin{tabular}{|c|c|c|c|c|c|c|c|}
\hline $\mathcal{S}_{L}$ & 0.251 & 0.361 & 0.527 & 0.919 & 1.551 & 3.254 & 6.201 \\
\hline $\mathcal{S}_{S}$ & 0.248 & 0.348 & 0.484 & 0.729 & 0.950 & 1.149 & 1.211 \\
\hline $\mathcal{S}_{L} / \mathcal{S}_{S}-1$ & 0.012 & 0.037 & 0.088 & 0.261 & 0.632 & 1.833 & 4.121 \\
\hline
\end{tabular}
\end{tabular}

Panel with $b=10 \%$

\begin{tabular}{|c|c|c|c|c|c|c|c|}
\hline $\mathcal{S}_{L}$ & 0.251 & 0.361 & 0.527 & 0.919 & 1.551 & 3.254 & 6.201 \\
\hline $\mathcal{S}_{S}$ & 0.247 & 0.341 & 0.462 & 0.641 & 0.734 & 0.699 & 0.595 \\
\hline $\mathcal{S}_{L} / \mathcal{S}_{S}-1$ & 0.019 & 0.058 & 0.141 & 0.435 & 1.111 & 3.653 & 9.424 \\
\hline
\end{tabular}

Panel with $b=14 \%$

\begin{tabular}{|c|c|c|c|c|c|c|c|}
\hline $\mathcal{S}_{L}$ & 0.251 & 0.361 & 0.527 & 0.919 & 1.551 & 3.254 & 6.201 \\
\hline $\mathcal{S}_{S}$ & 0.245 & 0.333 & 0.435 & 0.548 & 0.536 & 0.379 & 0.2443 \\
\hline $\mathcal{S}_{L} / \mathcal{S}_{S}-1$ & 0.028 & 0.085 & 0.210 & 0.678 & 1.892 & 7.577 & 24.384 \\
\hline
\end{tabular}


Table 2: Sharpe Ratios for a mean-reverting environment

This table displays the two Sharpe ratios $\mathcal{S}_{L}$ and $\mathcal{S}_{S}$ in the mean-reverting environment described in Section 5. In the calculation of the Sharpe ratios, $b$ is set to $6 \%$. The other parameters are: $\lambda=0.0226 \times 12, \sigma_{\theta}=0.0189 \times \sqrt{12}, \bar{\theta}=0.0788, r=0.0168$. We calculate the Sharpe ratios for four initial levels of $\theta_{0}: 0.35,0.10,0.0788,0.03$. The numbers in brackets are standard errors. The cases studied in the first three panels are not directly comparable to those in Table 1. Panel four gives the Sharpe ratios differences in a benchmark iid model which is comparable to the mean reverting model under consideration.

\begin{tabular}{|c|c|c|c|c|}
\hline year & $1 / 2$ & 1 & 2 & 5 \\
\hline \hline
\end{tabular}

Panel with $\theta_{0}=0.35$

\begin{tabular}{|c|c|c|c|c|}
\hline $\mathcal{S}_{L}$ & 0.2475 & 0.3502 & 0.4965 & 0.7973 \\
\hline $\mathcal{S}_{S}$ & 0.2335 & 0.3060 & 0.3188 & 0.0631 \\
\hline $\mathcal{S}_{L} / \mathcal{S}_{S}-1$ & 0.0599 & 0.1448 & 0.5573 & 11.6284 \\
& $(0.0358)$ & $(0.0272)$ & $(0.0278)$ & $(0.4489)$ \\
\hline
\end{tabular}

Panel with $\theta_{0}=0.10$

\begin{tabular}{|c|c|c|c|c|}
\hline $\mathcal{S}_{L}$ & 0.0756 & 0.1131 & 0.1752 & 0.3333 \\
\hline $\mathcal{S}_{S}$ & 0.0603 & 0.0550 & 0.0372 & 0.0088 \\
\hline $\mathcal{S}_{L} / \mathcal{S}_{S}-1$ & 1.2537 & 1.0574 & 3.7122 & 37.0467 \\
& $(0.0113)$ & $(0.0163)$ & $(0.0487)$ & $(1.5907)$ \\
\hline
\end{tabular}

Panel with $\theta_{0}=0.03$

\begin{tabular}{|c|c|c|c|c|}
\hline $\mathcal{S}_{L}$ & 0.0332 & 0.0587 & 0.1079 & 0.2477 \\
\hline $\mathcal{S}_{S}$ & 0.0227 & 0.0240 & 0.0196 & 0.0042 \\
\hline $\mathcal{S}_{L} / \mathcal{S}_{S}-1$ & 0.4605 & 1.4452 & 4.4940 & 57.9807 \\
& $(0.0062)$ & $(0.0132)$ & $(0.0509)$ & $(6.2139)$ \\
\hline
\end{tabular}

Panel for iid distribution $\left(\sigma_{\theta}=0\right.$ and $\left.\theta=\bar{\theta} \equiv 0.0788\right)$

\begin{tabular}{|c|c|c|c|c|}
\hline $\mathcal{S}_{L}$ & 0.0558 & 0.0789 & 0.1118 & 0.1776 \\
\hline $\mathcal{S}_{S}$ & 0.0544 & 0.0734 & 0.0941 & 0.1088 \\
\hline $\mathcal{S}_{L} / \mathcal{S}_{S}-1$ & 0.0243 & 0.0753 & 0.1880 & 0.6328 \\
\hline
\end{tabular}

Panel with $\theta_{0}=0.0788$

\begin{tabular}{|c|c|c|c|c|}
\hline $\mathcal{S}_{L}$ & 0.0619 & 0.0951 & 0.1525 & 0.3043 \\
\hline $\mathcal{S}_{S}$ & 0.0457 & 0.0420 & 0.0302 & 0.0070 \\
\hline $\mathcal{S}_{L} / \mathcal{S}_{S}-1$ & 0.3552 & 1.2659 & 4.0464 & 42.6018 \\
& $(0.0107)$ & $(0.0186)$ & $(0.0461)$ & $(2.5133)$ \\
\hline
\end{tabular}


Table 3: Frequency distribution of a $2 \times 2$ classification of the risk adjustment ratio and "Winner/Loser" variables.

The table displays a $2 \times 2$ contingency matrix for a simulated sample of 3000 funds which are run by a manager investing in an iid index and maximizing the one year Sharpe ratio. We simulate the sample after calibrating the short term interest rate from the 3-months treasury bill $(r=8.21 \%)$ and the index mean and variance from a value-weighted US equity index $\mu=16.02 \%, \sigma=16.73 \%$ during the period 1980-1991 (We used the data from the K. French web site). In the first panel (labeled "Median"), the funds are ranked with respect to the median value of the return $R$ during the same year. The second panel (labeled "Quartile"), the loser funds are the bottom quartile and the winner funds are the top quartile across funds during the same year. In each panel, the line "BHS" line reports the empirical findings in the base case scenario of Brown et al (1996) while the other lines report the contingency frequency table for the simulated sample for various level of targeted expected return $(b)$.

\begin{tabular}{|c|c||c|c|}
\hline \multicolumn{2}{|c||}{ Low Returns $R$ ("Losers") } & \multicolumn{2}{c|}{ High Returns $R$ ("Winners") } \\
\hline Low $R A R$ & High $R A R$ & Low $R A R$ & High $R A R$ \\
\hline
\end{tabular}

I) Median

\begin{tabular}{|c|c||c|c||c|c|}
\hline & $b=8.3 \%$ & $20.26 \%$ & $29.76 \%$ & $29.76 \%$ & $20.22 \%$ \\
& $b=9 \%$ & $19.99 \%$ & $30.02 \%$ & $30.02 \%$ & $19.97 \%$ \\
& $b=13 \%$ & $19.05 \%$ & $30.96 \%$ & $30.96 \%$ & $19.03 \%$ \\
& $b=16 \%$ & $18.23 \%$ & $31.79 \%$ & $31.79 \%$ & $18.19 \%$ \\
& $b=20 \%$ & $17.33 \%$ & $32.69 \%$ & $32.69 \%$ & $17.29 \%$ \\
& $b=25 \%$ & $16.46 \%$ & $33.55 \%$ & $33.55 \%$ & $16.44 \%$ \\
\hline BHS & & $22.46 \%$ & $27.46 \%$ & $27.46 \%$ & $22.62 \%$ \\
\hline
\end{tabular}

II) Quartile

\begin{tabular}{|c|c||c|c||c|c|}
\hline & $b=8.3 \%$ & $18.07 \%$ & $31.93 \%$ & $33.93 \%$ & $16.07 \%$ \\
& $b=9 \%$ & $17.80 \%$ & $32.20 \%$ & $34.33 \%$ & $15.67 \%$ \\
& $b=13 \%$ & $16.13 \%$ & $33.87 \%$ & $35.73 \%$ & $14.27 \%$ \\
& $b=16 \%$ & $14.73 \%$ & $35.27 \%$ & $36.60 \%$ & $13.40 \%$ \\
& $b=20 \%$ & $13.47 \%$ & $36.53 \%$ & $37.87 \%$ & $12.13 \%$ \\
& $b=25 \%$ & $12.33 \%$ & $37.67 \%$ & $38.80 \%$ & $11.20 \%$ \\
\hline BHS & & $22.63 \%$ & $27.25 \%$ & $28.63 \%$ & $21.49 \%$ \\
\hline
\end{tabular}




\section{References}

[1] Bajeux-Besnainou I. and R. Portait (1998): "Dynamic Asset Allocation in a MeanVariance Framework," Management Science, 44, N.11, 79-95.

[2] Barberis, N. (2000): "Investing for the Long Run when Returns are Predictable," Journal of Finance, 55, 225-264.

[3] Berk, J. and R. Green (2004): "Mutual Fund Flows and Performance in Rational Markets," Journal of Political Economy, 112, 1269-1295.

[4] Berkowitz, M. K. and Y. Kotowitz (1993): "Incentives and Efficiency in the Market for Management Services: A Study of Canadian Mutual Funds," Canadian Journal of Economics, 26, 850-866.

[5] Berkowitz, M. K. and Y. Kotowitz (2000): "Investor Risk Evaluation in the Determination of Management Incentives in the Mutual Fund Industry," Journal of Financial Markets, 3, 365-387.

[6] Bernardo, A. and O. Ledoit (2000): "Gain, Loss and Asset Pricing," Journal of Political Economy, 108, 144-172.

[7] Bielecki, T.R., Pliska, S.R., Jin H., and X.Y. Zhou (2005): "Continuous-Time MeanVariance Portfolio Selection with Bankruptcy Prohibition", Math. Finance 15, 213-244.

[8] Brown, K.C, Harlow, W.V. and L. T. Starks (1996): "Of Tournaments and Temptations: An Analysis of Managerial Incentives in the Mutual Fund Industry", Journal of Finance, 51, 85-110.

[9] Busse, J. A. (2001): "Another Look at Mutual Fund Tournaments," Journal of Financial and Quantitative Analysis, 36, 53-73.

[10] Cadenillas, A., Cvitanić, J. and F. Zapatero (2004): "Leverage Decision and Manager Compensation with Choice of Effort and Volatility," Journal of Financial Economics, 73, 71-92.

[11] Campbell, J. Y., L.M. Viceira (1999): "Consumption and Portfolio Decisions When Expected Returns are Time Varying," The Quarterly Journal of Economics, 114: 433495.

[12] Chevalier, J. and G. Ellison (1997): "Risk Taking by Mutual Funds as a Response to Incentives," Journal of Political Economy, 105, 11677-1120. 
[13] Chevalier, J. and G. Ellison (1999): "Career Concerns of Mutual Fund Managers," Quarterly Journal of Economics, 114, 389-432.

[14] Cuoco, D. and R. Kaniel (2003): "Equilibrium Prices in the Presence of Delegated Portfolio Management," working paper, Wharton.

[15] Cvitanić, J. and F. Zapatero (2004): Introduction to the Economics and Mathematics of Financial Markets. The MIT Press, Cambridge, Massachusetts.

[16] Goetzmann, W., J. Ingersoll, M. Spiegel, and I. Welch (2002): "Sharpening Sharpe Ratio," Yale working paper.

[17] Goetzmann, W., N. Peles (1997): "Cognitive Dissonance and Mutual Fund Investors," The Journal of Financial Research, 20, 145-158.

[18] Grinblatt, M. and S. Titman (1995): "Performance Evaluation," in Handboods in Operations Research and Management Science, Vol.9, 581-609.

[19] Huang, J., K.D. Wei and H. Yan (2004): "Participation Costs and the Sensitivity of Fund Flows to Past Performance," University of Texas working paper (forthcoming Journal of Finance).

[20] Karatzas,I. and S.E. Shreve, Brownian Motions and Stochastic Calculus, Berlin: Springer-Verlag, 1988.

[21] Koski, J.L. and Pontifff, J. (1999): "How are Derivatives Used? Evidence from the Mutual Fund Industry," Journal of Finance, 54, 791-816.

[22] Ippolito, R. (1992): "Consumer Reaction to Measures of Poor Quality: Evidence from the Mutual Fund Industry," Journal of Law and Economics, 35(1), 47-70.

[23] Korn, T. and S. Trautmann (1995): "Continuous-time Portfolio Optimization under Terminal Wealth Constraints", ZOR, 42, 69-93.

[24] Leland, E.H. (1999): "Beyond Mean-Variance: Performance Measurement in a Nonsymmetrical World," Financial Analysts Journal, vol 55.1, 27-36.

[25] Li, D. and W.L. Ng (2000): "Optimal Dynamic Portfolio Selection: Multiperiod MeanVariance Formulation", Math. Finance 10, 387-406.

[26] Lim A.E.B. (2003): "Quadratic Hedging and Mean-Variance Portfolio Selection in an Incomplete Market," Working paper, Berkeley (forthcoming in Mathematics of Operations Research). 
[27] Lim A and X.Y. Zhou. (2002): "Mean-Variance Portfolio Selection with Random Parameters in a Complete Market," Mathematics of Operations Research, vol 27.1, 101-20.

[28] Lintner, J. (1965): "The Valuation of Risky Assets and the Selection of Risky Investment in Stock Portfolios and Capital Budgets," Review of Economics and Statistics, 47, 13-37.

[29] D. Luenberger , Optimization by Vector Space Methods, New York, Wiley and Sons, 1979.

[30] Markowitz, H. (1952): "Portfolio Selection," Journal of Finance, 7, 77-91.

[31] Modigliani, F. and L. Modigliani (1997): "Risk-Adjusted Performance," Journal of Portfolio Management, 23, 45-54.

[32] Ou-Yang, H. (2003a): "Optimal Contracts in a Continuous-Time Delegated Portfolio Management Problem," Review of Financial Studies, 16, 173-208.

[33] Ou-Yang, H. (2003b): "An Equilibrium Model of Asset Pricing and Moral Hazard," Working paper, Duke University.

[34] Qiu, J. (2003): "Termination Risk, Multiple Managers and Mutual Fund Tournaments," European Finance Review, 7, 161-190.

[35] Sharpe, W. (1994): "The Sharpe Ratio," Journal of Portfolio Management, 21, 49-58.

[36] Sharpe, W. (1966): "Mutual Fund Performance," Journal of Business, 39, 119-138.

[37] Sharpe, W. (1964): "Capital Asset Prices: A Theory of Market Equilibrium under condition of Risk," Journal of Finance, 19, 425-442.

[38] Sirri, E.R. and Tufano, P. (1998): "Costly Search and Mutual Fund Flows," Journal of Finance, 53, 1589-1622.

[39] Spurgin, R. (2001): "How to Game Your Sharpe Ratio," Journal of Alternative Investments, 4, 38-46.

[40] Wachter, J. (2002): "Portfolio and Consumption Decisions under Mean-Reverting Returns: An Exact Solution for Complete Markets," Journal of Financial and Quantitative Analysis, 37, 63-91.

[41] Zhou, X.Y. and Li, D. (2000) : "Continuous Time Mean-Variance Portfolio Selection: A Stochastic LQ Framework," Applied Mathematics Optimization, 42, 19-33. 